\title{
On Kirchhoff's Model of Parabolic Type
}

\author{
Sudeep Kundu, Amiya K. Pani \\ Department of Mathematics \\ IIT Bombay, Powai, Mumbai-400076 (India) \\ Email: sudeep.kundu85@gmail.com, akp@math.iitb.ac.in \\ and \\ Morrakot Khebchareon \\ Department of Mathematics, Faculty of Science \\ Chiang Mai University, Chiang Mai-50200 (Thailand) \\ Email:mkhebcha@gmail.com.
}

November 6, 2018

\begin{abstract}
In this paper, existence of a strong global solution for all finite time is derived for the Kirchhoff's model of parabolic type. Based on exponential weight function, some new regularity results which reflect the exponential decay property are obtained for the exact solution. For the related dynamics, existence of a global attractor is shown to hold for the problem, when the nonhomogeneous forcing function is either independent of time or in $L^{\infty}\left(L^{2}\right)$. With finite element Galerkin method applied in spatial direction keeping time variable continuous, a semidiscrete scheme is analyzed and it is, further, established that the semi-discrete system has a global discrete attractor. Optimal error estimates in $L^{\infty}\left(H_{0}^{1}\right)$-norm are derived which are valid uniformly in time. Further, based on a Backward Euler method, a completely discrete scheme is developed and error estimates are derived. It is further observed that in case $f=0$ or $f=O\left(e^{-\gamma_{0} t}\right)$ with $\gamma_{0}>0$, the discrete solutions and also error estimates decay exponentially. Finally, some numerical experiments are discussed which confirm our theoretical findings.
\end{abstract}

\section{Inroduction.}

In this article, we consider the following nonlocal nonlinear boundary value problem of Kirchhoff's model of parabolic type: Find $u=u(x, t), x \in \Omega$ and $t>0$ which satisfies

$$
\begin{aligned}
u_{t}-\left(1+\|\nabla u(t)\|_{L^{2}(\Omega)}^{2}\right) \Delta u & =f & & \text { in } \Omega \times(0, \infty), \\
u(x, t) & =0 & & \text { on } \partial \Omega \times(0, \infty), \\
u(x, 0) & =u_{0} & & \text { in } \Omega,
\end{aligned}
$$

where $\Omega$ is a convex polygonal or polyhedral domain in $\mathbb{R}^{d}(d=2$ or 3$)$ with boundary $\partial \Omega$, $f=f(x, t)$ and $u_{0}$ are given functions in their respective domain of definitions. Here, $u_{t}=$ $\frac{\partial u}{\partial t}$ and $\|\cdot\|_{L^{2}(\Omega)}$ the $L^{2}$-norm. Such problem arises in the model describing the evolution of a population density subjected to a diffusion rate proportional to $\left(1+\|\nabla u(t)\|^{2}\right)$ with the forcing function $f$ representing the rate of supply. For details of the physical application and its complete mathematical modelling of such type of problems, we refer to 3 and [1]. More general nonlinear parabolic equations with nonlocal terms is of the form

$$
u_{t}-a\left(\|\nabla u\|_{L^{2}(\Omega)}^{2}\right) \Delta u=f(x, t), \quad(x, t) \in \Omega \times(0, \infty),
$$


where $a$ is a nonlinear nonlocal form in $u$, which includes the problem 1.1) are considered in the literature, see [5], 9], 4] and [2]. In these articles, the focus is on proving well-posedness and on the study of asymptotic behavior of solutions of the nonlocal problem (1.4)-(1.3) under various conditions on the nonlinearity.

In recent years, numerical approximation to the stationary problem of 1.1$)-(1.3)$ has been studied in [6] using $C^{0}$-conforming finite element method and optimal error estimates in $H^{1}$ are derived. However, there is hardly any result on the numerical approximations to (1.1)-(1.3). When the forcing function $f$ is either independent of time or is in $L^{\infty}\left(L^{2}\right)$, it plays a crucial role in the dynamics of this problem, therefore, in this paper, global existence of a unique strong solution to the problem (1.1)- 1.3 for all $t \in[0, T]$ with any finite positive $T>0$ is proved using Bubnov Galerkin method and compactness arguments. New regularity results using exponential weight function are also established. As a consequence, this problem admits the existence of a global attractor both in $L^{2}$ and $H_{0}^{1}$-spaces. When $C^{0}$ - conforming finite element method is applied to approximate the solution of (1.1) keeping time variable continuous, a semidiscrete scheme is derived and it is shown that the discrete problem has a discrete global attractor. Further, optimal priori error estimates in $L^{\infty}\left(H^{1}\right)$-norm are established which are even valid uniformly in time. Then based on backward Euler method, a discrete scheme is analyzed and it is, further, shown that the discrete problem has a solution using a variant of Brouwer fixed point argument. Moreover, optimal error estimates are derived. When either $f=0$ or $f=O\left(e^{-\gamma_{0} t}\right)$ with some $\gamma_{0}>0$, exponential decay property for the exact as well as the discrete solution and for error estimates is shown to hold. Finally some numerical experiments are conducted which confirm our theoretical results.

The main contributions of this article are to

- derive regularity results using exponential weight functions and establish global existence of a unique strong solution to problem (1.1)- 1.3 .

- prove optimal error estimates of the semidiscrete Galerkin approximation, which are valid uniformly in time and with right kind of regularity for the problem with convex polygonal or polyhedral domains.

- show the existence of a global attractor in both continuous and semidiscrete cases.

- prove exponential decay property for the exact solution, discrete solution and even for the error when the forcing function is either zero or of decaying exponentially in time.

- provide error analysis for the completely discrete scheme which is based on backward Euler method without using discrete Gronwall's inequality.

The rest of the article is organized as follows. Section 2 is devoted to the global existence and uniqueness of strong solution and new a priori bounds for the solutions of (1.1)- 1.3 are derived. Section 3 deals with error estimates for the semidiscrete solutions. Section 4 , focuses on the existence and uniqueness of the discrete solution and error estimates. Finally, in section 5 some numerical results are discussed to confirm theoretical results.

\section{Global Existence, Uniqueness and Regularity Results}

This section deals with weak formulation, existence of unique global solution and some regularity results. Denote by $H^{m}(\Omega)$ as the standard Sobolev spaces with norm $\|\cdot\|_{m}$.

Set $H_{0}^{1}(\Omega)=\left\{v \in H^{1}(\Omega): v=0 \quad\right.$ on $\left.\partial \Omega\right\}$. Let $H^{-1}$ be the dual space of $H_{0}^{1}(\Omega)$. The space $L^{p}([0, T] ; X) 1 \leq p \leq \infty$, consists of all strongly measurable functions $v:[0, T] \rightarrow X$ with norm

$$
\|v\|_{L^{p}([0, T] ; X)}:=\left(\int_{0}^{T}\|v(t)\|^{p} d t\right)^{\frac{1}{p}}<\infty \quad \text { for } \quad 1 \leq p<\infty,
$$

and

$$
\|v\|_{L^{\infty}([0, T] ; X)}:=\operatorname{esssup}_{0 \leq t \leq T}\|v(t)\|<\infty .
$$


The weak formulation related to the problem $1.1-1.3$ is to seek $v(t) \in H_{0}^{1}(\Omega), t>0$ such that

$$
\left(u_{t}, v\right)+\left(1+\|\nabla u\|_{L^{2}(\Omega)}^{2}\right)(\nabla u, \nabla v)=(f, v) \quad \forall v \in H_{0}^{1}(\Omega) \quad \text { with } u(0)=u_{0} .
$$

\subsection{A priori bounds}

This subsection focuses on a priori bounds for the problem 2.1) which are valid uniformly in time using exponential weight functions in time.

Lemma 2.1. Assume that $f \in L^{\infty}\left(H^{-1}\right)$ and $u_{0} \in L^{2}(\Omega)$. Then, there holds for $0<\alpha<\frac{\lambda_{1}}{2}$

$$
\begin{gathered}
\|u(t)\|^{2}+\beta e^{-2 \alpha t} \int_{0}^{t} e^{2 \alpha s}\|\nabla u(s)\|^{2} d s+2 e^{-2 \alpha t} \int_{0}^{t} e^{2 \alpha s}\|\nabla u(s)\|^{4} d s \\
\leq e^{-2 \alpha t}\left\|u_{0}\right\|^{2}+\frac{1}{2 \alpha}\|f\|_{L^{\infty}\left(H^{-1}\right)}^{2}\left(1-e^{-2 \alpha t}\right)=\widehat{K}_{0}(t) \\
\leq\left\|u_{0}\right\|^{2}+\frac{1}{2 \alpha}\|f\|_{L^{\infty}\left(H^{-1}\right)}^{2}=K_{0},
\end{gathered}
$$

where $\beta=\left(1-\frac{2 \alpha}{\lambda_{1}}\right)>0$, and $\lambda_{1}>0$ is the minimum eigenvalue of the Dirichlet eigenvalue problem for the Laplace operator.

Proof. Set $v=e^{2 \alpha t} u$ in 2.1 and obtain

$$
\begin{aligned}
\frac{1}{2} \frac{d}{d t}\left(e^{2 \alpha t}\|u(t)\|^{2}\right)-\alpha e^{2 \alpha t}\|u(t)\|^{2}+e^{2 \alpha t}\left(1+\|\nabla u(t)\|^{2}\right)\|\nabla u\|^{2} & \leq e^{2 \alpha t}\|f\|_{H^{-1}}\|\nabla u\| \\
& \leq \frac{1}{2} e^{2 \alpha t}\|f\|_{-1}^{2}+\frac{1}{2} e^{2 \alpha t}\|\nabla u\|^{2} .
\end{aligned}
$$

Apply Poincare's inequality : $\|\varphi\| \leq \frac{1}{\sqrt{\lambda_{1}}}\|\nabla \varphi\|$ for $\varphi \in H_{0}^{1}$, where $\lambda_{1}$ is the minimum eigenvalue of the Laplace operator with Dirichlet boundary condition. Thus, on integration with respect to time and using $a b \leq \frac{a^{2}}{2}+\frac{b^{2}}{2}$, we obtain

$$
\begin{aligned}
e^{2 \alpha t}\|u(t)\|^{2}+\left(1-\frac{2 \alpha}{\lambda_{1}}\right) \int_{0}^{t} e^{2 \alpha s}\|\nabla u(s)\|^{2} d s & +2 \int_{0}^{t} e^{2 \alpha s}\|\nabla u(s)\|^{4} d s \\
& \leq\left\|u_{0}\right\|^{2}+\frac{1}{2 \alpha}\left(e^{2 \alpha t}-1\right)\|f\|_{L^{\infty}\left(H^{-1}\right)}^{2} .
\end{aligned}
$$

Since $\alpha$ can be chosen so that $0<\alpha<\frac{\lambda_{1}}{2}$ and $\beta=\left(1-\frac{2 \alpha}{\lambda_{1}}\right)>0$. Then, it follows that

$$
\begin{aligned}
\|u(t)\|^{2}+\beta e^{-2 \alpha t} \int_{0}^{t} e^{2 \alpha s}\|\nabla u(s)\|^{2} d s & +2 e^{-2 \alpha t} \int_{0}^{t} e^{2 \alpha s}\|\nabla u(s)\|^{4} d s \\
& \leq e^{-2 \alpha t}\left\|u_{0}\right\|^{2}+\frac{1}{2 \alpha}\|f\|_{L^{\infty}\left(H^{-1}\right)}^{2}\left(1-e^{-2 \alpha t}\right),
\end{aligned}
$$

and this concludes the rest of the proof.

Remark 2.1. If $f \in L^{\infty}\left(L^{2}\right)$, then rewrite using Poincare's inequality

$$
\begin{aligned}
e^{2 \alpha t}(f, u) \leq e^{2 \alpha t}\|f\|_{L^{\infty}\left(L^{2}\right)}\|u\| & \leq \frac{e^{2 \alpha t}}{\sqrt{\lambda_{1}}}\|f\|_{L^{\infty}\left(L^{2}\right)}\|\nabla u\| \\
& \leq \frac{e^{2 \alpha t}}{2 \lambda_{1}}\|f\|_{L^{\infty}\left(L^{2}\right)}^{2}+\frac{1}{2}\|\nabla u\|^{2}
\end{aligned}
$$

Following the part of Lemma 2.1, it now follows that

$$
\|u(t)\| \leq e^{-2 \alpha t}\left\|u_{0}\right\|^{2}+\frac{1}{2 \lambda_{1} \alpha}\|f\|_{L^{\infty}\left(L^{2}\right)}^{2}\left(1-e^{-2 \alpha t}\right) .
$$


Lemma 2.2. Let $u_{0} \in H_{0}^{1}(\Omega)$ and $f \in L^{\infty}\left(L^{2}\right)$. Then for $0<\alpha<\frac{\lambda_{1}}{2}$, there holds:

$$
\begin{gathered}
\|\nabla u(t)\|^{2}+\beta e^{-2 \alpha t} \int_{0}^{t} e^{2 \alpha s}\|\Delta u(s)\|^{2} d s+2 e^{-2 \alpha t} \int_{0}^{t} e^{2 \alpha s}\|\nabla u(s)\|^{2}\|\Delta u(s)\|^{2} d s \\
\leq e^{-2 \alpha t}\left\|\nabla u_{0}\right\|^{2}+\frac{1}{2 \alpha}\|f\|_{L^{\infty}\left(L^{2}\right)}^{2}\left(1-e^{-2 \alpha t}\right)=\widehat{K}_{1}(t) \\
\leq\left\|\nabla u_{0}\right\|^{2}+\frac{1}{2 \alpha}\|f\|_{L^{\infty}\left(L^{2}\right)}^{2}=K_{1} .
\end{gathered}
$$

Proof. Forming $L^{2}$ - inner product between (1.1) and $-e^{2 \alpha t} \Delta u$ yields

$$
\begin{aligned}
\frac{1}{2} \frac{d}{d t}\left(e^{2 \alpha t}\|\nabla u(t)\|^{2}\right)-\alpha e^{2 \alpha t}\|\nabla u(t)\|^{2} & +\left(1+\|\nabla u\|^{2}\right) e^{2 \alpha t}\|\Delta u(t)\|^{2} \\
& =-e^{2 \alpha t}(f, \Delta u) \\
& \leq \frac{1}{2} e^{2 \alpha t}\|f\|^{2}+\frac{1}{2} e^{2 \alpha t}\|\Delta u\|^{2} .
\end{aligned}
$$

Using Poincare's inequality, it follows using integration with respect to time that

$$
\begin{aligned}
e^{2 \alpha t}\|\nabla u(t)\|^{2}+\left(1-\frac{2 \alpha}{\lambda_{1}}\right) \int_{0}^{t} e^{2 \alpha s}\|\Delta u(s)\|^{2} d s & +2 \int_{0}^{t} e^{2 \alpha s}\|\nabla u(s)\|^{2}\|\Delta u(s)\|^{2} d s \\
& \leq\left\|\nabla u_{0}\right\|^{2}+\frac{1}{2 \alpha}\|f\|_{L^{\infty}\left(L^{2}\right)}^{2}\left(e^{2 \alpha t}-1\right) .
\end{aligned}
$$

Multiplying by $e^{-2 \alpha t}$ and with $\beta=\left(1-\frac{2 \alpha}{\lambda_{1}}\right)>0$, it completes the rest of the proof.

Lemma 2.3. Let $u_{0} \in H_{0}^{1}(\Omega)$ and $f \in L^{\infty}\left(L^{2}\right)$. Then for $0<\alpha<\frac{\lambda_{1}}{2}$, there holds:

$$
\begin{aligned}
2 e^{-2 \alpha t} \int_{0}^{t} e^{2 \alpha s}\left\|u_{s}\right\|^{2} d s+\left(2+\|\nabla u(t)\|^{2}\right)\|\nabla u(t)\|^{2} & \\
& \leq\left(2+\left\|\nabla u_{0}\right\|^{2}\right)\left\|\nabla u_{0}\right\|^{2} e^{-2 \alpha t}+\frac{1}{\alpha}\|f\|_{L^{\infty}\left(L^{2}\right)}^{2}\left(1-e^{-2 \alpha t}\right)+\alpha\left(1+\frac{4}{\beta}\right) \widehat{K}_{0}(t)=\widehat{K}_{3}(t) \\
& \leq\left(2+\left\|\nabla u_{0}\right\|^{2}\right)\left\|\nabla u_{0}\right\|^{2}+\frac{1}{\alpha}\|f\|_{L^{\infty}\left(L^{2}\right)}^{2}+\alpha\left(1+\frac{4}{\beta}\right) K_{0}=K_{3} .
\end{aligned}
$$

Proof. Set $v=u_{t}$ in 2.1 and obtain

$$
\left\|u_{t}\right\|^{2}+\frac{1}{4} \frac{d}{d t}\left(\left(2+\|\nabla u\|^{2}\right)\|\nabla u\|^{2}\right) \leq \frac{1}{2}\|f\|^{2}+\frac{1}{2}\left\|u_{t}\right\|^{2},
$$

and hence,

$$
\left\|u_{t}\right\|^{2}+\frac{1}{2} \frac{d}{d t}\left(2+\|\nabla u\|^{2}\right)\|\nabla u\|^{2} \leq\|f\|^{2} .
$$

Multiplying by $2 e^{2 \alpha t}$ and then, rewrite it as

$$
2 e^{2 \alpha t}\left\|u_{t}\right\|^{2}+\frac{d}{d t}\left(e^{2 \alpha t}\left(2+\|\nabla u\|^{2}\right)\|\nabla u\|^{2}\right) \leq 2 e^{2 \alpha t}\|f\|^{2}+2 \alpha e^{2 \alpha t}\left(2+\|\nabla u\|^{2}\right)\|\nabla u\|^{2} .
$$

Thus, on integration with respect time from 0 to $t$ and then, multiplying the resulting inequality by $e^{-2 \alpha t}$ to obtain

$$
\begin{aligned}
2 e^{-2 \alpha t} \int_{0}^{t} e^{2 \alpha s}\left\|u_{s}\right\|^{2} d s+ & \left(2+\|\nabla u\|^{2}\right)\|\nabla u\|^{2} \\
\leq & \left(2+\left\|\nabla u_{0}\right\|^{2}\right)\left\|\nabla u_{0}\right\|^{2} e^{-2 \alpha t}+\frac{1}{\alpha}\|f\|_{L^{\infty}\left(L^{2}\right)}^{2}\left(1-e^{-2 \alpha t}\right) \\
& +2 \alpha e^{-2 \alpha t} \int_{0}^{t} e^{2 \alpha s}\left(2+\|\nabla u\|^{2}\right)\|\nabla u\|^{2} d s .
\end{aligned}
$$


Using Lemma 2.1, we note that

$$
2 \alpha e^{-2 \alpha t} \int_{0}^{t} e^{2 \alpha s}\left(2+\|\nabla u\|^{2}\right)\|\nabla u\|^{2} d s \leq \alpha\left(1+\frac{4}{\beta}\right) \widehat{K}_{0}(t) .
$$

Thus, we arrive at

$$
\begin{aligned}
2 e^{-2 \alpha t} \int_{0}^{t} e^{2 \alpha s}\left\|u_{s}\right\|^{2} d s+ & \left(2+\|\nabla u(t)\|^{2}\right)\|\nabla u(t)\|^{2} \\
& \leq\left(2+\left\|\nabla u_{0}\right\|^{2}\right)\left\|\nabla u_{0}\right\|^{2} e^{-2 \alpha t}+\frac{1}{\alpha}\|f\|_{L^{\infty}\left(L^{2}\right)}^{2}\left(1-e^{-2 \alpha t}\right) \\
& +\alpha\left(1+\frac{4}{\beta}\right) \widehat{K}_{0}(t)=\widehat{K}_{3}(t) \\
& \leq\left(2+\left\|\nabla u_{0}\right\|^{2}\right)\left\|\nabla u_{0}\right\|^{2}+\frac{1}{\alpha}\|f\|_{L^{\infty}\left(L^{2}\right)}^{2}+\alpha\left(1+\frac{4}{\beta}\right) K_{0}=K_{3} .
\end{aligned}
$$

This concludes the rest of the proof.

Lemma 2.4. Let $u_{0} \in H^{2} \cap H_{0}^{1}(\Omega), f \in L^{\infty}\left(L^{2}\right)$ and $f_{t} \in L^{\infty}\left(H^{-1}\right)$. Then there holds

$$
\begin{aligned}
& \left\|u_{t}(t)\right\|^{2}+e^{-2 \alpha t} \int_{0}^{t} e^{2 \alpha s}\left(\beta+2\|\nabla u\|^{2}\right)\left\|\nabla u_{s}\right\|^{2} d s+4 e^{-2 \alpha t} \int_{0}^{t} e^{2 \alpha s}\left(\nabla u_{s}, \nabla u\right)^{2} d s \\
& \leq e^{-2 \alpha t}\left(\left(1+\left\|\nabla u_{0}\right\|^{2}\right)\left\|u_{0}\right\|_{H^{2}}+\left\|f_{0}\right\|\right)^{2}+e^{-2 \alpha t} \int_{0}^{t} e^{2 \alpha s}\left\|f_{s}\right\|_{-1}^{2} d s .
\end{aligned}
$$

Proof. Differentiating of 2.1] with respect to time yields

$$
\left(u_{t t}, v\right)+\left(\left(1+\|\nabla u\|^{2}\right) \nabla u_{t}, \nabla v\right)+\left(2\left(\nabla u_{t}, \nabla u\right) \nabla u, \nabla v\right)=\left(f_{t}, v\right) .
$$

Substitute $v=u_{t}$ in 2.5 to obtain

$$
\frac{1}{2} \frac{d}{d t}\left\|u_{t}\right\|^{2}+\left(1+\|\nabla u\|^{2}\right)\left\|\nabla u_{t}\right\|^{2}+2\left(\nabla u_{t}, \nabla u\right)^{2}=\left(f_{t}, u_{t}\right) .
$$

Multiplying (2.6) by $2 e^{2 \alpha t}, \alpha>0$ and using Poincare's inequality it follows that

$$
\begin{array}{r}
\frac{d}{d t}\left(e^{2 \alpha t}\left\|u_{t}\right\|^{2}\right)+e^{2 \alpha t}\left(1-\frac{2 \alpha}{\lambda_{1}}\right)\left\|\nabla u_{t}\right\|^{2}+2 e^{2 \alpha t}\left(1+\|\nabla u\|^{2}\right)\left\|\nabla u_{t}\right\|^{2}+4 e^{2 \alpha t}\left(\nabla u_{t}, \nabla u\right)^{2} \\
\leq e^{2 \alpha t}\left\|f_{t}\right\|_{-1}^{2} .
\end{array}
$$

Integrating with respect to time from 0 to $t$ and multiplying the resulting inequality by $e^{-2 \alpha t}$ to obtain

$$
\begin{aligned}
& \left\|u_{t}(t)\right\|^{2}+e^{-2 \alpha t} \int_{0}^{t} e^{2 \alpha s}\left(\beta+2\|\nabla u\|^{2}\right)\left\|\nabla u_{s}\right\|^{2} d s+4 e^{-2 \alpha t} \int_{0}^{t} e^{2 \alpha s}\left(\nabla u_{s}, \nabla u\right)^{2} d s \\
& \leq e^{-2 \alpha t}\left\|u_{t}(0)\right\|^{2}+e^{-2 \alpha t} \int_{0}^{t} e^{2 \alpha s}\left\|f_{s}\right\|_{-1}^{2} d s \\
& \leq e^{-2 \alpha t}\left(\left(1+\left\|\nabla u_{0}\right\|^{2}\right)\left\|u_{0}\right\|_{H^{2}}+\left\|f_{0}\right\|\right)^{2}+e^{-2 \alpha t} \int_{0}^{t} e^{2 \alpha s}\left\|f_{s}\right\|_{-1}^{2} d s .
\end{aligned}
$$

This completes the rest of the proof.

Lemma 2.5. Let $u_{0} \in H^{2} \cap H_{0}^{1}(\Omega), f \in L^{\infty}\left(L^{2}\right)$ and $f_{t} \in L^{\infty}\left(H^{-1}\right)$. Then, there holds:

$$
\begin{array}{r}
\left(1+\|\nabla u\|^{2}\right)\|\Delta u\|^{2} \leq 2\|f\|_{L^{\infty}\left(L^{2}\right)}^{2}+2 e^{-2 \alpha t}\left(\left(\left(1+\left\|\nabla u_{0}\right\|^{2}\right)\left\|u_{0}\right\|_{H^{2}}+\left\|f_{0}\right\|\right)^{2}+\right. \\
\left.\int_{0}^{t} e^{2 \alpha s}\left\|f_{s}\right\|_{-1}^{2} d s\right) .
\end{array}
$$


Proof. Substitute $v=-\Delta u$ in the Weak formulation (2.1) to obtain

$$
\left(1+\|\nabla u\|^{2}\right)\|\Delta u\|^{2}=-(f, \Delta u)+\left(u_{t}, \Delta u\right) .
$$

Using Young's inequality, we bound

$$
\begin{aligned}
\left(1+\|\nabla u\|^{2}\right)\|\Delta u\|^{2} & \leq\|f\|_{L^{\infty}\left(L^{2}\right)}^{2}+\left\|u_{t}\right\|_{L^{\infty}\left(L^{2}\right)}^{2}+\frac{1}{2}\|\Delta u\|^{2} \\
& \leq\|f\|_{L^{\infty}\left(L^{2}\right)}^{2}+\left\|u_{t}\right\|_{L^{\infty}\left(L^{2}\right)}^{2}+\frac{1}{2}\left(1+\|\nabla u\|^{2}\right)\|\Delta u\|^{2} .
\end{aligned}
$$

Therefore, we arrive at

$$
\left(1+\|\nabla u\|^{2}\right)\|\Delta u\|^{2} \leq 2\|f\|_{L^{\infty}\left(L^{2}\right)}^{2}+2\left\|u_{t}\right\|_{L^{\infty}\left(L^{2}\right)}^{2} .
$$

From Lemma 2.4 applying the bound of $\left\|u_{t}\right\|$ we obtain bound for $\|\Delta u\|$. This completes the proof.

Since $\Omega$ is a convex polygonal bounded domain, hence $\|u\|_{H^{2}}^{2} \leq C_{R}\|\Delta u\|^{2}$. Now from Lemmas 2.42 .5 , it follows that

$\|u(t)\|_{H^{2}}^{2} \leq 2 C_{R}\left(\|f\|_{L^{\infty}\left(L^{2}\right)}^{2}+e^{-2 \alpha t}\left(\left(1+\left\|\nabla u_{0}\right\|^{2}\right)^{2}\left\|u_{0}\right\|_{H^{2}}^{2}+\left\|f_{0}\right\|^{2}\right)+e^{-2 \alpha t} \int_{0}^{t} e^{2 \alpha s}\left\|f_{s}\right\|_{-1}^{2} d s\right)$.

Lemma 2.6. Let $u_{0} \in H^{3}, f \in L^{\infty}\left(L^{2}\right)$ and $f_{t} \in L^{\infty}\left(H^{-1}\right)$. Then, there holds

$$
\begin{aligned}
\left\|\nabla u_{t}\right\|^{2}+ & e^{-2 \alpha t} \int_{0}^{t} e^{2 \alpha s}\left(1+\|\nabla u\|^{2}\right)\left\|\Delta u_{t}(s)\right\|^{2} d s \\
& \leq C\left(e^{-2 \alpha t}\left\|u_{0}\right\|_{H^{3}}^{2}+e^{-2 \alpha t}\left(\left(1+\left\|\nabla u_{0}\right\|^{2}\right)^{2}\left\|u_{0}\right\|_{H^{2}}^{2}+\left\|f_{0}\right\|^{2}\right)\right. \\
& \left.+e^{-2 \alpha t} \int_{0}^{t} e^{2 \alpha s}\left\|f_{s}\right\|_{-1}^{2} d s\right) .
\end{aligned}
$$

Proof. Differentiating of 2.1] with respect to time yields

$$
\left(u_{t t}, v\right)-\left(\left(1+\|\nabla u\|^{2}\right) \Delta u_{t}, v\right)-\left(2\left(\nabla u_{t}, \nabla u\right) \Delta u, v\right)=\left(f_{t}, v\right) .
$$

Substitute $v=-\Delta u_{t}$ in 2.9 to obtain

$$
\frac{d}{d t}\left\|\nabla u_{t}\right\|^{2}+2\left(1+\|\nabla u\|^{2}\right)\left\|\Delta u_{t}\right\|^{2}=2\left(f_{t},-\Delta u_{t}\right)+4\left(\left(u_{t},-\Delta u\right) \Delta u,-\Delta u_{t}\right) .
$$

On multiplying 2.10 by $e^{2 \alpha t}, \alpha>0$ and using Young's inequality, it follows that

$$
\begin{aligned}
\frac{d}{d t}\left(e^{2 \alpha t}\left\|\nabla u_{t}\right\|^{2}\right) & +2 e^{2 \alpha t}\left(1+\|\nabla u\|^{2}\right)\left\|\Delta u_{t}\right\|^{2} \\
& \leq 2 e^{2 \alpha t}\left\|f_{t}\right\|^{2}+\frac{1}{2} e^{2 \alpha t}\left\|\Delta u_{t}\right\|^{2}+8 e^{2 \alpha t}\left\|u_{t}\right\|^{2}\|\Delta u\|^{4} \\
& +\frac{1}{2} e^{2 \alpha t}\left\|\Delta u_{t}\right\|^{2}+2 \alpha e^{2 \alpha t}\left\|\nabla u_{t}\right\|^{2}
\end{aligned}
$$

Now using Poincare's inequality, we find that

$$
\begin{aligned}
\frac{d}{d t}\left(e^{2 \alpha t}\left\|\nabla u_{t}\right\|^{2}\right) & +e^{2 \alpha t}\left(1+\|\nabla u\|^{2}\right)\left\|\Delta u_{t}\right\|^{2} \\
& \leq 2 e^{2 \alpha t}\left\|f_{t}\right\|^{2}+8 e^{2 \alpha t}\left\|u_{t}\right\|^{2}\|\Delta u\|^{4}+2 \alpha e^{2 \alpha t}\left\|\nabla u_{t}\right\|^{2} .
\end{aligned}
$$


Integrating with respect to time from 0 to $t$ and multiplying the resulting inequality by $e^{-2 \alpha t}$ to obtain

$$
\begin{aligned}
& \left\|\nabla u_{t}\right\|^{2}+e^{-2 \alpha t} \int_{0}^{t} e^{2 \alpha s}\left(1+\|\nabla u\|^{2}\right)\left\|\Delta u_{t}(s)\right\|^{2} d s \\
& \leq e^{-2 \alpha t}\left\|\nabla u_{t}(0)\right\|^{2}+2 e^{-2 \alpha t} \int_{0}^{t} e^{2 \alpha s}\left\|f_{s}\right\|^{2} d s \\
& +8 e^{-2 \alpha t} \int_{0}^{t} e^{2 \alpha s}\left\|u_{t}\right\|^{2}\|\Delta u\|^{4} d s+2 \alpha e^{-2 \alpha t} \int_{0}^{t} e^{2 \alpha s}\left\|\nabla u_{t}(s)\right\|^{2} d s .
\end{aligned}
$$

Therefore by Lemma 2.4, we arrive at

$$
\begin{aligned}
\left\|\nabla u_{t}\right\|^{2}+ & e^{-2 \alpha t} \int_{0}^{t} e^{2 \alpha s}\left(1+\|\nabla u\|^{2}\right)\left\|\Delta u_{t}(s)\right\|^{2} d s \\
& \leq C\left(e^{-2 \alpha t}\left\|u_{0}\right\|_{H^{3}}^{2}+e^{-2 \alpha t}\left(\left(1+\left\|\nabla u_{0}\right\|^{2}\right)^{2}\left\|u_{0}\right\|_{H^{2}}^{2}+\left\|f_{0}\right\|^{2}\right)\right. \\
& \left.+e^{-2 \alpha t} \int_{0}^{t} e^{2 \alpha s}\left\|f_{s}\right\|_{-1}^{2} d s\right) .
\end{aligned}
$$

This completes the rest of the proof.

Lemma 2.7. Let $u_{0} \in H^{3}, f \in L^{\infty}\left(L^{2}\right)$ and $f_{t} \in L^{\infty}\left(H^{-1}\right)$. Then, the following result holds

$$
\begin{aligned}
& e^{-2 \alpha t} \int_{0}^{t} e^{2 \alpha s}\left\|u_{t t}(s)\right\|^{2} d s+\left(1+\|\nabla u(t)\|^{2}\right)\left\|\nabla u_{t}\right\|^{2} \\
& \quad \leq e^{-2 \alpha t}\left\|u_{0}\right\|_{H^{3}}^{2}+C\left(K_{1}\right)\left(e^{-2 \alpha t}\left(\left(1+\left\|\nabla u_{0}\right\|^{2}\right)^{2}\left\|u_{0}\right\|_{H^{2}}^{2}+\left\|f_{0}\right\|^{2}\right)+e^{-2 \alpha t} \int_{0}^{t} e^{2 \alpha s}\left\|f_{s}\right\|_{-1}^{2} d s\right) .
\end{aligned}
$$

Proof. Differentiating of 2.1 with respect to time yields

$$
\left(u_{t t}, v\right)+\left(\left(1+\|\nabla u\|^{2}\right) \nabla u_{t}, \nabla v\right)+\left(2\left(\nabla u_{t}, \nabla u\right) \nabla u, \nabla v\right)=\left(f_{t}, v\right) .
$$

Substitute $v=u_{t t}$ in 2.15 to obtain

$$
\left\|u_{t t}\right\|^{2}+\frac{1}{2}\left(1+\|\nabla u\|^{2}\right) \frac{d}{d t}\left\|\nabla u_{t}\right\|^{2}-2\left(\left(\nabla u_{t}, \nabla u\right) \Delta u, u_{t t}\right)=\left(f_{t}, u_{t t}\right) .
$$

On multiplying (2.16) by $e^{2 \alpha t}, \alpha>0$ and rewriting it as

$$
\begin{aligned}
e^{2 \alpha t}\left\|u_{t t}\right\|^{2}+\frac{1}{2} \frac{d}{d t}\left(e^{2 \alpha t}\left(1+\|\nabla u\|^{2}\right)\left\|\nabla u_{t}\right\|^{2}\right) & =e^{2 \alpha t}\left(f_{t}, u_{t t}\right)+2 e^{2 \alpha t}\left(\left(\nabla u_{t}, \nabla u\right) \Delta u, u_{t t}\right) \\
& +e^{2 \alpha t}\left(\nabla u_{t}, \nabla u\right)\left\|\nabla u_{t}\right\|^{2}+\alpha e^{2 \alpha t}\left(1+\|\nabla u\|^{2}\right)\left\|\nabla u_{t}\right\|^{2} .
\end{aligned}
$$

Now using Young's inequality for the first two terms in the right hand side and rewriting it as

$$
\begin{aligned}
e^{2 \alpha t}\left\|u_{t t}\right\|^{2}+ & \frac{d}{d t}\left(e^{2 \alpha t}\left(1+\|\nabla u\|^{2}\right)\left\|\nabla u_{t}\right\|^{2}\right) \\
& \leq 2 e^{2 \alpha t}\left\|f_{t}\right\|^{2}+e^{2 \alpha t}\left(8\|\nabla u\|^{2}\|\Delta u\|^{2}+2\|\nabla u\|\left\|\nabla u_{t}\right\|+2 \alpha\left(1+\|\nabla u\|^{2}\right)\right)\left\|\nabla u_{t}\right\|^{2} .
\end{aligned}
$$

Integrating with respect to time from 0 to $t$ and multiplying the resulting inequality by $e^{-2 \alpha t}$ to obtain

$$
\begin{aligned}
& e^{-2 \alpha t} \int_{0}^{t} e^{2 \alpha s}\left\|u_{t t}(s)\right\|^{2} d s+\left(1+\|\nabla u(t)\|^{2}\right)\left\|\nabla u_{t}\right\|^{2} \\
& \leq e^{-2 \alpha t}\left(1+\|\nabla u(0)\|^{2}\right)\left\|\nabla u_{t}(0)\right\|^{2}+2 e^{-2 \alpha t} \int_{0}^{t} e^{2 \alpha s}\left\|f_{s}\right\|^{2} d s \\
& \quad+e^{-2 \alpha t} \int_{0}^{t} e^{2 \alpha s}\left(8\|\nabla u\|^{2}\|\Delta u\|^{2}+2\|\nabla u\|\left\|\nabla u_{t}(s)\right\|+2 \alpha\left(1+\|\nabla u\|^{2}\right)\right)\left\|\nabla u_{t}(s)\right\|^{2} d s .
\end{aligned}
$$


The term inside the bracket of the third term in the right hand side is bounded by Lemmas 2.2 , 2.5 and 2.6. Therefore the third term in the right hand side is bounded by the lemma 2.4. Altogether, we obtain

$$
e^{-2 \alpha t} \int_{0}^{t} e^{2 \alpha s}\left\|u_{t t}(s)\right\|^{2} d s+\left(1+\|\nabla u(t)\|^{2}\right)\left\|\nabla u_{t}\right\|^{2}
$$

$$
\leq e^{-2 \alpha t}\left\|u_{0}\right\|_{H^{3}}^{2}+C\left(K_{1}\right)\left(e^{-2 \alpha t}\left(\left(1+\left\|\nabla u_{0}\right\|^{2}\right)^{2}\left\|u_{0}\right\|_{H^{2}}^{2}+\left\|f_{0}\right\|^{2}\right)+e^{-2 \alpha t} \int_{0}^{t} e^{2 \alpha s}\left\|f_{s}\right\|_{-1}^{2} d s\right) .
$$

This completes the rest of the proof.

Remark 2.2. $\quad$ 1. When $f=0$, then we obtain

$$
\|u(t)\|,\|\nabla u(t)\|=O\left(e^{-\alpha t}\right) \quad \text { and } \quad \tau^{\frac{1}{2}}\left(\left\|u_{t}(t)\right\|+\|u(t)\|_{H^{2}}\right)=O\left(e^{-\alpha t}\right) .
$$

Hence, we derive exponential decay property.

2. When $f \in L^{\infty}\left(L^{2}\right)$ with $\|f\|_{L^{\infty}\left(L^{2}\right)}=O\left(e^{-\gamma_{0} t}\right)$, then for $\alpha_{0}=\min \left(\alpha, \gamma_{0}\right)$; the solution decays exponentially with order $O\left(e^{-\alpha_{0} t}\right)$.

3. If $f \in L^{\infty}\left(L^{2}\right)$, we obtain regularity results proved in Lemmas 2.1 2.7 are valid uniformly in time for $\alpha=0$.

\subsection{Existence and Uniqueness of strong solution}

Before, proving existence and uniqueness of a strong solution, we first prove the following monotonicty property for our subsequent use.

Lemma 2.8. For $u$ and $v \in H_{0}^{1}$, there holds

$$
\left(\left(1+\|\nabla u\|^{2}\right) \nabla u-\left(1+\|\nabla v\|^{2}\right) \nabla v, \nabla(u-v)\right) \geq\|\nabla(u-v)\|^{2} .
$$

Proof. Note that

$$
\begin{aligned}
\left(\left(1+\|\nabla u\|^{2}\right) \nabla u-\left(1+\|\nabla v\|^{2}\right) \nabla v, \nabla(u-v)\right) & =\|\nabla(u-v)\|^{2}+\left(\|\nabla u\|^{2} \nabla u-\|\nabla v\|^{2} \nabla v, \nabla(u-v)\right) \\
& =\|\nabla(u-v)\|^{2}+\left(\|\nabla u\|^{2} \nabla(u-v), \nabla(u-v)\right) \\
& +\left(\left(\|\nabla u\|^{2}-\|\nabla v\|^{2}\right) \nabla v, \nabla(u-v)\right) \\
& =\|\nabla(u-v)\|^{2}+\|\nabla u\|^{2}\|\nabla(u-v)\|^{2} \\
& +\left(\|\nabla u\|^{2}-\|\nabla v\|^{2}\right)(\nabla v, \nabla(u-v)) .
\end{aligned}
$$

Now the term $\left(\|\nabla u\|^{2}-\|\nabla v\|^{2}\right)(\nabla v, \nabla(u-v))$ can be written as

$$
\begin{aligned}
& \left(\|\nabla u\|^{2}-\|\nabla v\|^{2}\right)(\nabla v, \nabla(u-v)) \\
= & \left(\|\nabla u\|^{2}-\|\nabla v\|^{2}\right)(\nabla(u+v), \nabla(u-v))-\left(\|\nabla u\|^{2}-\|\nabla v\|^{2}\right)(\nabla u, \nabla(u-v)) \\
\geq & \left(\|\nabla u\|^{2}-\|\nabla v\|^{2}\right)^{2}-\left(\|\nabla u\|^{2}-\|\nabla v\|^{2}\right)\|\nabla u\|\|\nabla(u-v)\| \\
\geq & \left(\|\nabla u\|^{2}-\|\nabla v\|^{2}\right)^{2}-\frac{1}{2}\left(\|\nabla u\|^{2}-\|\nabla v\|^{2}\right)^{2}-\frac{1}{2}\|\nabla u\|^{2}\|\nabla(u-v)\|^{2} \\
= & \frac{1}{2}\left(\|\nabla u\|^{2}-\|\nabla v\|^{2}\right)^{2}-\frac{1}{2}\|\nabla u\|^{2}\|\nabla(u-v)\|^{2} .
\end{aligned}
$$

Therefore,

$$
\begin{aligned}
\left(\left(1+\|\nabla u\|^{2}\right) \nabla u-\left(1+\|\nabla v\|^{2}\right) \nabla v, \nabla(u-v)\right) & \geq\|\nabla(u-v)\|^{2}+\frac{1}{2}\left(\|\nabla u\|^{2}-\|\nabla v\|^{2}\right)^{2} \\
& +\frac{1}{2}\|\nabla u\|^{2}\|\nabla(u-v)\|^{2} \geq\|\nabla(u-v)\|^{2} .
\end{aligned}
$$

This completes the rest of the proof. 
Theorem 2.1. Suppose that $u_{0} \in H_{0}^{1}(\Omega)$ and $f \in L^{\infty}\left(L^{2}\right)$. Then for any finite $T>0$, the problem (1.1)-1.3 admits a unique global strong solution $u$ for $t \in(0, T]$ satisfying

$$
u \in C\left([0, T], H_{0}^{1}\right) \cap L^{2}\left([0, T], H^{2}\right), u_{t} \in L^{2}\left([0, T], L^{2}\right) .
$$

Proof. For a proof of existence, one can apply Bubnov-Galerkin method and compactness arguments of Lions in a standard way, see also [9].

For uniqueness, we prove it by contradiction. Assume contrary, then there exist two distinct solutions $u_{1}$ and $u_{2}$ of the problem (1.1) satisfying

$$
\left(u_{i t}, v\right)+\left(\left(1+\left\|\nabla u_{i}\right\|^{2}\right) \nabla u_{i}, \nabla v\right)=(f, v), i=1,2 .
$$

With $w=u_{1}-u_{2}, w$ now satisfies

$$
\left(w_{t}, v\right)+\left(\left(1+\left\|\nabla u_{1}\right\|^{2}\right) \nabla u_{1}-\left(1+\left\|\nabla u_{2}\right\|^{2}\right) \nabla u_{2}, \nabla v\right)=0
$$

Substitute $v=u_{1}-u_{2}=w$ to obtain

$$
\left(w_{t}, w\right)+\left(\left(1+\left\|\nabla u_{1}\right\|^{2}\right) \nabla u_{1}-\left(1+\left\|\nabla u_{2}\right\|^{2}\right) \nabla u_{2}, \nabla\left(u_{1}-u_{2}\right)\right)=0
$$

Using monotonicity property given in Lemma 2.8 , we observe that

$$
\left(\left(1+\left\|\nabla u_{1}\right\|^{2}\right) \nabla u_{1}-\left(1+\left\|\nabla u_{2}\right\|^{2}\right) \nabla u_{2}, \nabla\left(u_{1}-u_{2}\right)\right) \geq\left\|\nabla\left(u_{1}-u_{2}\right)\right\|^{2}=\|\nabla w\|^{2} \geq 0 .
$$

Consequently,

$$
\frac{d}{d t}\|w\|^{2} \leq 0
$$

Since $w(0)=0$, it follows that $w=0$ which leads to a contradiction. Hence, the solution is unique. This completes the rest of the proof.

As a consequence of Lemmas 2.1 and 2.2, we obtain the following results: From 2.3 , we note that the ball $B_{\rho_{0}}(0)$ in $L^{2}(\Omega)$ is absorbing in $L^{2}(\Omega)$ with $\rho_{0}=\frac{1}{\sqrt{\alpha \lambda_{1}}}\|f\|_{L^{\infty}\left(L^{2}\right)}$. Specially, for any $R>0$, there exists $t_{0}=t_{0}\left(R, \rho_{0}\right)>0$ such that for $t \geq t_{0}=\frac{1}{2 \alpha} \log \left(\frac{2 R^{2}-\rho_{0}^{2}}{\rho_{0}^{2}}\right)$

$$
B_{R}(0) \subset B_{\rho_{0}}(0) .
$$

To provide a quick sketch of its proof, note that for any $R>0$ with $u_{0} \in B_{R}(0)$,

$$
\|u(t)\| \leq e^{-2 \alpha t} R^{2}+\frac{1}{2} \rho_{0}{ }^{2}\left(1-e^{-2 \alpha t}\right)=e^{-2 \alpha t}\left(R^{2}-\frac{1}{2} \rho_{0}{ }^{2}\right)+\frac{1}{2} \rho_{0}{ }^{2} \leq \rho_{0}{ }^{2},
$$

provided $e^{-2 \alpha t}\left(R^{2}-\frac{1}{2} \rho_{0}^{2}\right) \leq \frac{1}{2} \rho_{0}^{2}$, that is, $e^{2 \alpha t} \geq \frac{2 R^{2}-\rho_{0}^{2}}{\rho_{0}^{2}}$. Now taking $\log$ both sides, it follows that, $t \geq \frac{1}{2 \alpha} \log \left(\frac{2 R^{2}-\rho_{0}{ }^{2}}{\rho_{0}{ }^{2}}\right)=t_{0}$. Hence, $B_{\rho_{0}}(0)$ is an absorbing ball in $L^{2}(\Omega)$. Similarly from 2.4 in Lemma 2.2. it follows that for any $R>0$ and $u_{0} \in B_{R}(0) \subset H_{0}^{1}$,

$$
u(t) \leq \rho_{1} \quad \text { for } t \geq t_{1}=\frac{1}{2 \alpha} \log \left(\frac{2 R^{2}-\rho_{1}^{2}}{\rho_{1}^{2}}\right),
$$

where $\rho_{1}=\frac{1}{\sqrt{\alpha}}\|f\|_{L^{\infty}\left(L^{2}\right)}$. Therefore, $B_{\rho_{1}}(0)$ is an absorbing set in $H_{0}^{1}(\Omega)$ for the equation (2.1). Thus, we have the following result

Theorem 2.2. The problem 2.1)-2.4 admits a global attractor in $L^{2}$ as well in $H_{0}^{1}$. 


\section{Semidiscrete Galerkin Method}

This section deals with semidiscrete Galerkin approximation keeping time variable continuous and proves optimal error estimates.

Given a regular triangulation $\mathcal{T}_{h}$ of $\bar{\Omega}$, let $h_{K}=\operatorname{diam}(K)$ for all $K \in \mathcal{T}_{h}$ and $h=\max _{K \in \mathcal{T}_{h}} h_{K}$ Set

$$
V_{h}=\left\{v_{h} \in C^{0}(\bar{\Omega}):\left.v_{h}\right|_{K} \in \mathcal{P}_{1}(K) \quad \forall \quad K \in \mathcal{T}_{h} \text { with } v_{h}=0 \text { on } \partial \Omega\right\} .
$$

Under an additional assumption that the family of triangulation $\mathcal{T}_{h}$ is quasi-uniform, the following inverse inequality holds

$$
\|\nabla \chi\| \leq C h^{-1}\|\chi\| \quad \forall \chi \in V_{h} .
$$

Now the semidiscrete approximation $u_{h}(t)$ of 2.1 is to find $u_{h}(t) \in V_{h}$ for $t>0$ such that

$$
\left(u_{h t}, \chi\right)+\left(1+\left\|\nabla u_{h}\right\|_{L^{2}(\Omega)}^{2}\right)\left(\nabla u_{h}, \nabla \chi\right)=(f, \chi) \quad \forall \chi \in V_{h}
$$

with $u_{h}(0)=u_{0 h} \in V_{h}$ to be defined later.

Theorem 3.1. For any $u_{0 h} \in V_{h}$, there exists a unique solution $u_{h} \in C^{1}\left([0, \infty] ; V_{h}\right)$ satisfying (3.1).

Proof. Since $V_{h}$ is finite dimensional, (3.1) leads to a a system of nonlinear ODE's. An appeal to the Picard's theorem yields the existence of a unique solution $u_{h}(t)$ locally, that is, there exists $t=t^{*}>0$ such that 3.1 has a unique solution $u_{h}(t)$ for $t \in\left(0, t^{*}\right)$.

For global existence, we use continuation argument provided $\left\|u_{h}(t)\right\|$ is bounded for all $t>0$. Now choose $\chi=u_{h}$ in 3.1 to obtain as in Lemma 2.1 with $0<\alpha<\frac{\lambda_{1}}{2}$

$$
\begin{aligned}
\left\|u_{h}(t)\right\|^{2}+\min (\beta, 2) e^{-2 \alpha t} & \int_{0}^{t}\left(\left\|\nabla u_{h}(s)\right\|^{2}+\left\|\nabla u_{h}(s)\right\|^{4}\right) d s \\
& \leq e^{-2 \alpha t}\left\|u_{0 h}\right\|^{2}+\frac{1}{2 \alpha \lambda_{1}}\|f\|_{L^{\infty}\left(L^{2}\right)}^{2}\left(1-e^{-2 \alpha t}\right),
\end{aligned}
$$

where $\beta=\left(1-\frac{2 \alpha}{\lambda_{1}}\right)>0$. Note $u_{0 h}$ an approximation of $u_{0}$ in $V_{h}$ and $\left\|u_{0 h}\right\|$ can be made bounded by $\left\|u_{0}\right\|$. The result on global existence of a unique solution $u_{h}(t)$ of (3.1) now follows for all $t>0$. This completes the rest of the proof.

As a consequence, the following result holds as in the continuous case.

Proposition 3.1. There exists a bounded absorbing set $B_{R}(0)$ in $V_{h}$ for (3.1), that is, for $R>0$ and $u_{0 h} \in B_{R}(0)$, there exists $t=t_{0}\left(\left\|u_{0 h}\right\|\right)$ such that for $t \geq t_{0}, u_{h}(t) \in B_{\rho_{0}}(0)$, where $\rho_{0}=$ $\frac{1}{\sqrt{\alpha \lambda_{1}}}\|f\|_{L^{\infty}\left(L^{2}\right)}$.

Thus, as in continuous case, the following theorem holds.

Theorem 3.2. The equation (3.1) has a global attractor $\mathcal{A}_{h}$ which attracts bounded set in $V_{h}$.

\subsection{A priori bounds}

We now introduce discrete Laplacian $\Delta_{h}: V_{h} \longrightarrow V_{h}$ by

$$
\left(-\Delta_{h} v_{h}, w_{h}\right)=\left(\nabla v_{h}, \nabla w_{h}\right) \quad \forall v_{h}, w_{h} \in V_{h} .
$$


Lemma 3.1. Let $u_{0 h}$ is an approximation of $u_{0}$ and $f \in L^{\infty}\left(L^{2}\right)$. Then for $0<\alpha<\frac{\lambda_{1}}{2}$, there holds:

$$
\begin{aligned}
\left\|\nabla u_{h}(t)\right\|^{2}+\beta e^{-2 \alpha t} \int_{0}^{t} e^{2 \alpha s}\left\|\Delta_{h} u_{h}(s)\right\|^{2} d s & +2 e^{-2 \alpha t} \int_{0}^{t} e^{2 \alpha s}\left\|\nabla u_{h}(s)\right\|^{2}\left\|\Delta_{h} u_{h}(s)\right\|^{2} d s \\
& \leq e^{-2 \alpha t}\left\|\nabla u_{0 h}\right\|^{2}+\|f\|^{2}\left(1-e^{-2 \alpha t}\right)=\hat{K}_{4}(t) \\
& \leq\left\|\nabla u_{0}\right\|^{2}+\frac{1}{2 \alpha}\|f\|_{L^{\infty}\left(L^{2}\right)}^{2}=K_{1} .
\end{aligned}
$$

Proof. Proof is similar to the proof of Lemma 2.2 .

Lemma 3.2. Let $u_{0 h}$ is an approximation of $u_{0}, u_{0} \in H_{0}^{1}(\Omega)$ and $f \in L^{\infty}\left(L^{2}\right)$. Then there holds:

$$
\begin{aligned}
2 e^{-2 \alpha t} \int_{0}^{t} e^{2 \alpha s}\left\|u_{h s}\right\|^{2} d s+\left(2+\left\|\nabla u_{h}(t)\right\|^{2}\right)\left\|\nabla u_{h}(t)\right\|^{2} \\
\quad \leq\left(2+\left\|\nabla u_{h 0}\right\|^{2}\right)\left\|\nabla u_{0 h}\right\|^{2} e^{-2 \alpha t}+\frac{1}{\alpha}\|f\|_{L^{\infty}\left(L^{2}\right)}^{2}\left(1-e^{-2 \alpha t}\right)+\alpha\left(1+\frac{4}{\beta}\right) \widehat{K}_{0}(t)=\widehat{K}_{5}(t) \\
\quad \leq\left(2+\left\|\nabla u_{0 h}\right\|^{2}\right)\left\|\nabla u_{0 h}\right\|^{2}+\frac{1}{\alpha}\|f\|_{L^{\infty}\left(L^{2}\right)}^{2}+\alpha\left(1+\frac{4}{\beta}\right) K_{0}=K_{5} .
\end{aligned}
$$

Proof. Proof is similar to the proof of the Lemma 2.3

Lemma 3.3. Let $u_{0 h}$ is an approximation of $u_{0}, f \in L^{\infty}\left(L^{2}\right)$ and $f_{t} \in L^{2}\left(H^{-1}\right)$. Then there holds:

$\tau(t)\left\|u_{h t}(t)\right\|^{2}+e^{-2 \alpha t} \int_{0}^{t} \tau(s) e^{2 \alpha s}\left(\beta+2\left\|\nabla u_{h}\right\|^{2}\right)\left\|\nabla u_{h s}\right\|^{2} d s+4 e^{-2 \alpha t} \int_{0}^{t} \tau(s) e^{2 \alpha s}\left(\nabla u_{h s}, \nabla u_{h}\right)^{2} d s$ $\leq e^{-2 \alpha t} \int_{0}^{t} e^{2 \alpha s}\left\|f_{s}\right\|_{-1}^{2} d s+K_{5}$

where $\tau(t)=\min (t, 1)$.

Proof. Differentiating of 3.1 with respect to time yields

$$
\left(u_{h t t}, v\right)+\left(\left(1+\left\|\nabla u_{h}\right\|^{2}\right) \nabla u_{h t}, \nabla v\right)+\left(2\left(\nabla u_{h t}, \nabla u_{h}\right) \nabla u_{h}, \nabla v\right)=\left(f_{t}, v\right)
$$

Substitute $v=u_{h t}$ in 3.4 to obtain

$$
\frac{1}{2} \frac{d}{d t}\left\|u_{h t}\right\|^{2}+\left(1+\left\|\nabla u_{h}\right\|^{2}\right)\left\|\nabla u_{h t}\right\|^{2}+2\left(\nabla u_{h t}, \nabla u_{h}\right)^{2}=\left(f_{t}, u_{h t}\right) .
$$

Set $\tau(t)=\min (t, 1)$ and multiplying 3.5 by $2 \tau e^{2 \alpha t}, \alpha>0$ and using Poincare's inequality it follows that

$$
\begin{aligned}
\frac{d}{d t}\left(\tau e^{2 \alpha t}\left\|u_{h t}\right\|^{2}\right)+\tau(t) e^{2 \alpha t}\left(1-\frac{2 \alpha}{\lambda_{1}}\right) & \left\|\nabla u_{h t}\right\|^{2}+ \\
& 2 \tau(t) e^{2 \alpha t}\left(1+\left\|\nabla u_{h}\right\|^{2}\right)\left\|\nabla u_{h t}\right\|^{2}+4 \tau(t) e^{2 \alpha t}\left(\nabla u_{h t}, \nabla u_{h}\right)^{2} \\
& \leq e^{2 \alpha t}\left\|f_{t}\right\|_{-1}^{2}+e^{2 \alpha t}\left\|u_{h t}\right\|^{2} .
\end{aligned}
$$

Integrating with respect to time from 0 to $t$ and multiplying the resulting inequality by $e^{-2 \alpha t}$ to obtain

$\tau(t)\left\|u_{h t}(t)\right\|^{2}+e^{-2 \alpha t} \int_{0}^{t} \tau(s) e^{2 \alpha s}\left(\beta+2\left\|\nabla u_{h}\right\|^{2}\right)\left\|\nabla u_{h s}\right\|^{2} d s+4 e^{-2 \alpha t} \int_{0}^{t} \tau(s) e^{2 \alpha s}\left(\nabla u_{h s}, \nabla u_{h}\right)^{2} d s$

$$
\leq e^{-2 \alpha t} \int_{0}^{t} e^{2 \alpha s}\left\|f_{s}\right\|_{-1}^{2} d s+e^{-2 \alpha t} \int_{0}^{t} e^{2 \alpha s}\left\|u_{h s}\right\|^{2} d s .
$$

Now using Lemma 3.2 the second term in the right hand side is bounded and hence, this completes the rest of the proof. 
Lemma 3.4. Let $u_{0 h}$ is an approximation of $u_{0}, u_{0} \in H_{0}^{1}(\Omega), f \in L^{\infty}\left(L^{2}\right)$, and $f_{t} \in L^{2}\left(H^{-1}\right)$. Then, there holds:

$$
\begin{aligned}
\tau(t)\left(1+\left\|\nabla u_{h}(t)\right\|^{2}\right)\left\|\Delta u_{h}(t)\right\|^{2} & \leq 2 \tau\|f(t)\|_{L^{\infty}\left(L^{2}\right)}^{2}+2 \tau\left\|u_{h t}(t)\right\|_{L^{\infty}\left(L^{2}\right)}^{2} \\
& \leq 2\|f(t)\|_{L^{\infty}\left(L^{2}\right)}^{2}+2 e^{-2 \alpha t} \int_{0}^{t} e^{2 \alpha s}\left\|f_{s}\right\|_{-1}^{2} d s+K_{5} .
\end{aligned}
$$

Proof. Substitute $v=-\Delta_{h} u_{h}$ in the weak formulation (3.1) to obtain

$$
\left(1+\left\|\nabla u_{h}\right\|^{2}\right)\left\|\Delta_{h} u_{h}\right\|^{2}=-\left(f, \Delta_{h} u_{h}\right)+\left(u_{t}, \Delta_{h} u_{h}\right),
$$

and using Young's inequality, and $(3.3)$,we now bound

$$
\begin{aligned}
\left(1+\left\|\nabla u_{h}\right\|^{2}\right)\left\|\Delta_{h} u_{h}\right\|^{2} & \leq\|f\|_{L^{\infty}\left(L^{2}\right)}^{2}+\left\|u_{h t}\right\|_{L^{\infty}\left(L^{2}\right)}^{2}+\frac{1}{2}\left\|\Delta_{h} u_{h}\right\|^{2} \\
& \leq\|f\|_{L^{\infty}\left(L^{2}\right)}^{2}+\left\|u_{h t}\right\|_{L^{\infty}\left(L^{2}\right)}^{2}+\frac{1}{2}\left(1+\left\|\nabla u_{h}\right\|^{2}\right)\left\|\Delta_{h} u_{h}\right\|^{2} .
\end{aligned}
$$

Therefore, multiplying by the resulting inequality by $\tau$ it follows that

$$
\tau\left(1+\left\|\nabla u_{h}\right\|^{2}\right)\left\|\Delta_{h} u_{h}\right\|^{2} \leq 2 \tau\|f\|_{L^{\infty}\left(L^{2}\right)}^{2}+2 \tau\left\|u_{h t}\right\|_{L^{\infty}\left(L^{2}\right)}^{2} .
$$

From Lemma 3.3 applying the bound of $\tau\left\|u_{t}\right\|^{2}$, we obtain bound for $\left\|\Delta_{h} u_{h}\right\|$. This completes the rest of the proof.

\subsection{A priori Error estimates}

This subsection focuses on error estimates of the semidiscrete Galerkin approximation.

Let $\widetilde{u}_{h}(t) \in V_{h}$ be the Ritz-projection of $u(t) \in H_{0}^{1}(\Omega)$ defined by

$$
\left(\nabla\left(u-\widetilde{u}_{h}\right), \nabla \chi\right)=0 \forall \chi \in V_{h} .
$$

For each $t>0, \widetilde{u}_{h}(t) \in V_{h}$ is welldefined for a given $u(t)$. With $\eta=u-\widetilde{u}_{h}$, the following error estimates hold:

$$
\|\eta\|_{j} \leq C h^{\min (2, m)-j}\|u\|_{m}, \text { and }\left\|\eta_{t}\right\| \leq C h^{\min (2, m)-j}\left\|u_{t}\right\|_{m}, \quad j=0,1 \text { and } m=1,2
$$

For a proof, refer to Thomee [8]. Now split $u-u_{h}=\left(u-\widetilde{u}_{h}\right)-\left(u_{h}-\widetilde{u}_{h}\right):=\eta-\theta$

Since estimates of $\eta$ are known, it is enough to estimate $\theta$. Using (2.1), (3.1) and (3.8), we arrive at an equation in $\theta$ as

$$
\begin{aligned}
\left(\theta_{t}, \chi\right)+\left(\left(1+\left\|\nabla u_{h}\right\|^{2}\right) \nabla u_{h}-\left(1+\left\|\nabla \widetilde{u}_{h}\right\|^{2}\right)\right. & \left.\nabla \widetilde{u}_{h}, \nabla \chi\right)=\left(\eta_{t}, \chi\right) \\
& +\left(\left(1+\|\nabla u\|^{2}\right) \nabla u-\left(1+\left\|\nabla \widetilde{u}_{h}\right\|^{2}\right) \nabla \widetilde{u}_{h}, \nabla \chi\right)
\end{aligned}
$$

Theorem 3.3. Let $u_{0} \in H_{0}^{1}(\Omega), f \in L^{\infty}\left(L^{2}\right)$ and $f_{t} \in L^{2}\left(H^{-1}\right)$. Then there holds:

$$
\begin{aligned}
& \|\theta(t)\|^{2}+\beta e^{-2 \alpha t} \int_{0}^{t} e^{2 \alpha s}\|\nabla \theta(s)\|^{2} d s \\
& \leq C\left(K_{1}\right) h^{2} e^{-2 \alpha t}\left(\left\|\nabla u_{0}\right\|^{2}+\int_{0}^{t} e^{2 \alpha s}\left\|f_{s}\right\|_{-1}^{2} d s+\left(\left(1+\left\|\nabla u_{0}\right\|^{2}\right)^{2}\left\|u_{0}\right\|_{H^{2}}^{2}+\left\|f_{0}\right\|^{2}\right)\right),
\end{aligned}
$$

where $K_{1}$ depends on $\left\|\nabla u_{0}\right\|$ and $\|f\|_{L^{\infty}\left(L^{2}\right)}$. 
Proof. Set $\chi=\theta$ in 3.10 and use also the monotonicity property to obtain

$$
\begin{aligned}
\frac{1}{2} \frac{d}{d t}\|\theta(t)\|^{2}+\|\nabla \theta\|^{2} & \leq\left\|\eta_{t}\right\|\|\theta\|+\left(\|\nabla u\|^{2}-\left\|\nabla \widetilde{u}_{h}\right\|^{2}\right)\|\nabla u\|\|\nabla \theta\| \\
& \leq\left(\frac{1}{\left.\sqrt{(} \lambda_{1}\right)}\left\|\eta_{t}\right\|+\left(\|\nabla u\|+\left\|\nabla \widetilde{u}_{h}\right\|\right)\|\nabla \eta\|\|\nabla u\|\right)\|\nabla \theta\| \\
& \leq \frac{1}{\lambda_{1}}\left\|\eta_{t}\right\|^{2}+\left(\|\nabla u\|+\left\|\nabla \widetilde{u}_{h}\right\|\right)^{2}\|\nabla \eta\|^{2}\|\nabla u\|^{2}+\frac{1}{2}\|\nabla \theta\|^{2}
\end{aligned}
$$

Here, we have used Poincare inequality and Youngs inequality. Multiply by $2 e^{2 \alpha t}, \alpha>0$ and rewrite it as

$$
\begin{aligned}
\frac{d}{d t}\left(e^{2 \alpha t}\|\theta(t)\|^{2}\right)- & 2 \alpha e^{2 \alpha t}\|\theta\|^{2}+e^{2 \alpha t}\|\nabla \theta\|^{2} \\
& \leq \frac{1}{\lambda_{1}} e^{2 \alpha t}\left\|\eta_{t}\right\|^{2}+2\left(\|\nabla u\|^{2}+\left\|\nabla \widetilde{u}_{h}\right\|^{2}\right)\|\nabla u\|^{2}\left(e^{2 \alpha t}\|\nabla \eta\|^{2}\right) .
\end{aligned}
$$

Using Poincare's inequality $\|\theta\|^{2} \leq \frac{1}{\lambda_{1}}\|\nabla \theta\|^{2}$ with $\alpha>0$ such that $\beta=\left(1-\frac{2 \alpha}{\lambda_{1}}\right)>0$ and integrating with respect to $t$ from 0 to $t$ to obtain

$$
\begin{array}{r}
\|\theta(t)\|^{2}+\beta e^{-2 \alpha t} \int_{0}^{t} e^{2 \alpha s}\|\nabla \theta(s)\|^{2} d s \leq e^{-2 \alpha t}\|\theta(0)\|^{2}+\frac{1}{\lambda_{1}} e^{-2 \alpha t} \int_{0}^{t} e^{2 \alpha s}\left\|\eta_{s}\right\|^{2} d s \\
+2 e^{-2 \alpha t} \int_{0}^{t}\left(\|\nabla u\|^{2}+\left\|\nabla \widetilde{u}_{h}\right\|^{2}\right)\|\nabla u\|^{2} e^{2 \alpha s}\|\nabla \eta(s)\|^{2} d s .
\end{array}
$$

With a choice $u_{h 0}=\widetilde{u}_{h}(0), \theta(0)=0$. But with $u_{h 0}=P_{h} u_{0}$ or $u_{h 0}=I_{h} u_{0}$, where $P_{h}$ and $I_{h}$, respectively, are $L^{2}$ - projection and interpolant onto $V_{h}$, then

$$
\|\theta(0)\| \leq\left\|u_{h 0}-u_{0}\right\|+\left\|u_{0}-\widetilde{u}_{h}(0)\right\| \leq C h\left\|\nabla u_{0}\right\| .
$$

Then using regularity result in Lemma 2.2 we arrive at

$$
\begin{aligned}
\|\theta(t)\|^{2}+ & \beta e^{-2 \alpha t} \int_{0}^{t} e^{2 \alpha s}\|\nabla \theta(s)\|^{2} d s \\
& \leq C\left(K_{1}\right) h^{2} e^{-2 \alpha t}\left(\left\|\nabla u_{0}\right\|^{2}+\int_{0}^{t} e^{2 \alpha s}\left\|\nabla u_{s}\right\|^{2}+\int_{0}^{t} e^{2 \alpha s}\|\Delta u(s)\|^{2}\right) .
\end{aligned}
$$

An application of Lemma 2.2, 2.4 yields the final result. This completes the rest of the proof.

Since, the estimate $\|\nabla \eta\| \leq C h\|u\|_{2}$ is known, it is enough to prove the estimate of $\nabla \theta$.

Theorem 3.4. Let $u_{0} \in H^{2} \cap H_{0}^{1}(\Omega), f \in L^{\infty}\left(L^{2}\right)$ and $f_{t} \in L^{2}\left(H^{-1}\right)$. Then, there holds:

$$
\begin{aligned}
e^{-2 \alpha t} \int_{0}^{t} \tau(s) e^{2 \alpha s}\left\|\theta_{s}\right\|^{2} d s & +\tau(t)\left(1+\left\|\nabla u_{h}\right\|^{2}\right)\|\nabla \theta(t)\|^{2} \\
& \left.\leq C\left(K_{1}, K_{5}\right) h^{2} e^{-2 \alpha t}\left(\left(1+\| \nabla u_{0}\right) \|^{2}\right)^{2}\left\|u_{0}\right\|_{H^{2}}^{2}+\left\|f_{0}\right\|^{2}+\int_{0}^{t} e^{2 \alpha s}\left\|f_{s}\right\|_{-1}^{2} d s\right) .
\end{aligned}
$$

Proof. Setting $\chi=\theta_{t}$ in 3.10 , it follows that

$$
\begin{aligned}
\left(\theta_{t}, \theta_{t}\right)+ & \left(\left(1+\left\|\nabla u_{h}\right\|^{2}\right) \nabla u_{h}-\left(1+\left\|\nabla \widetilde{u}_{h}\right\|^{2}\right) \nabla \widetilde{u}_{h}, \nabla \theta_{t}\right)=\left(\eta_{t}, \theta_{t}\right) \\
& \left(\left(1+\|\nabla u\|^{2}\right) \nabla u-\left(1+\left\|\nabla \widetilde{u}_{h}\right\|^{2}\right) \nabla \widetilde{u}_{h}, \nabla \theta_{t}\right)
\end{aligned}
$$


Now multiplying by $e^{2 \alpha t}, \alpha>0$ and applying Ritz projection the equation 3.13 can be written as

$$
\begin{gathered}
\left.e^{2 \alpha t}\left\|\theta_{t}\right\|^{2}+e^{2 \alpha t}\left(\nabla \theta, \nabla \theta_{t}\right)+e^{2 \alpha t}\left(\left\|\nabla u_{h}\right\|^{2} \nabla u_{h}-\left\|\nabla \widetilde{u}_{h}\right\|^{2} \nabla \widetilde{u}_{h}\right), \nabla \theta_{t}\right) \\
=e^{2 \alpha t}\left(\eta_{t}, \theta_{t}\right)+e^{2 \alpha t}\left(\|\nabla u\|^{2} \nabla u-\left\|\nabla \widetilde{u}_{h}\right\|^{2} \nabla \widetilde{u}_{h}, \nabla \theta_{t}\right)
\end{gathered}
$$

Now rewrite it as

$$
\begin{aligned}
e^{2 \alpha t}\left\|\theta_{t}\right\|^{2}+ & \frac{1}{2} \frac{d}{d t}\left(e^{2 \alpha t}\|\nabla \theta\|^{2}\right)-\alpha e^{2 \alpha t}\|\nabla \theta\|^{2}+e^{2 \alpha t}\left(\left\|\nabla u_{h}\right\|^{2} \nabla u_{h}-\left\|\nabla \widetilde{u}_{h}\right\|^{2} \nabla \widetilde{u}_{h}, \nabla \theta_{t}\right) \\
& =e^{2 \alpha t}\left(\eta_{t}, \theta_{t}\right)+e^{2 \alpha t}\left(\|\nabla u\|^{2} \nabla u-\left\|\nabla \widetilde{u}_{h}\right\|^{2} \nabla \widetilde{u}_{h}, \nabla \theta_{t}\right) .
\end{aligned}
$$

A use of the Ritz projection shows

$$
\begin{aligned}
e^{2 \alpha t}\left(\|\nabla u\|^{2} \nabla u-\left\|\nabla \widetilde{u}_{h}\right\|^{2} \nabla \widetilde{u}_{h}, \nabla \theta_{t}\right) & =e^{2 \alpha t}\left(\left(\|\nabla u\|^{2}-\left\|\nabla \widetilde{u}_{h}\right\|^{2}\right) \nabla \widetilde{u}_{h}, \nabla \theta_{t}\right) \\
& =e^{2 \alpha t}\left(\left(\|\nabla u\|^{2}-\left\|\nabla \widetilde{u}_{h}\right\|^{2}\right) \nabla u, \nabla \theta_{t}\right) \\
& -e^{2 \alpha t}\left(\left(\|\nabla u\|^{2}-\left\|\nabla \widetilde{u}_{h}\right\|\right) \nabla\left(u-\widetilde{u}_{h}\right), \nabla \theta_{t}\right) \\
& =-e^{2 \alpha t}\left(\left(\|\nabla u\|^{2}-\left\|\nabla \widetilde{u}_{h}\right\|^{2}\right) \Delta u, \theta_{t}\right) \\
& \leq e^{2 \alpha t}\left(\|\nabla u\|+\left\|\nabla \widetilde{u}_{h}\right\|\right)\|\nabla \eta\|\|\Delta u\|\left\|\theta_{t}\right\| .
\end{aligned}
$$

For the third term on the left-hand side of 3.14 , rewrite it as

$$
\begin{aligned}
e^{2 \alpha t}\left(\left\|\nabla u_{h}\right\|^{2} \nabla u_{h}-\left\|\nabla \widetilde{u}_{h}\right\|^{2} \nabla \widetilde{u}_{h}, \nabla \theta_{t}\right) & =\frac{1}{2} \frac{d}{d t}\left(\|\nabla \theta\|^{2}\right) e^{2 \alpha t}\left\|\nabla u_{h}\right\|^{2} \\
& -e^{2 \alpha t}\left(\left(\left\|\nabla u_{h}\right\|^{2}-\left\|\nabla \widetilde{u}_{h}\right\|^{2}\right) \nabla\left(u-\widetilde{u}_{h}\right), \nabla \theta_{t}\right) \\
& +e^{2 \alpha t}\left(\left(\left\|\nabla u_{h}\right\|^{2}-\left\|\nabla \widetilde{u}_{h}\right\|^{2}\right) \nabla u, \nabla \theta_{t}\right) \\
& =\frac{1}{2} \frac{d}{d t}\left(\|\nabla \theta\|^{2}\right) e^{2 \alpha t}\left\|\nabla u_{h}\right\|^{2} \\
& -e^{2 \alpha t}\left(\left(\left\|\nabla u_{h}\right\|^{2}-\left\|\nabla \widetilde{u}_{h}\right\|^{2}\right) \Delta u, \theta_{t}\right) .
\end{aligned}
$$

Similarly,

$$
\begin{aligned}
& \frac{1}{2} \frac{d}{d t}\left(e^{2 \alpha t}\|\nabla \theta\|^{2}\right)+\frac{1}{2} \frac{d}{d t}\left(\|\nabla \theta\|^{2}\right) e^{2 \alpha t}\left\|\nabla u_{h}\right\|^{2} \\
& =\frac{1}{2} \frac{d}{d t}\left(\left(1+\left\|\nabla u_{h}\right\|^{2}\right) e^{2 \alpha t}\|\nabla \theta\|^{2}\right)-e^{2 \alpha t}\|\nabla \theta\|^{2}\left(u_{h t},-\Delta_{h} u_{h}\right)-\alpha e^{2 \alpha t}\left\|\nabla u_{h}\right\|^{2}\|\nabla \theta\|^{2} .
\end{aligned}
$$

Substitute 3.15, (3.16, 3.17 in 3.14 to obtain

$$
\begin{aligned}
e^{2 \alpha t}\left\|\theta_{t}\right\|^{2}+\frac{1}{2} \frac{d}{d t}\left(\left(1+\left\|\nabla u_{h}\right\|^{2}\right) e^{2 \alpha t}\|\nabla \theta\|^{2}\right) & \leq e^{2 \alpha t}\left\|\eta_{t}\right\|\left\|\theta_{t}\right\| \\
& +e^{2 \alpha t}\left(\left(\left\|\nabla u_{h}\right\|^{2}-\left\|\nabla \widetilde{u}_{h}\right\|^{2}\right) \Delta u, \theta_{t}\right) \\
& +e^{2 \alpha t}\left(\|\nabla u\|+\left\|\nabla \widetilde{u}_{h}\right\|\right)\|\nabla \eta\|\|\Delta u\|\left\|\theta_{t}\right\| \\
& +e^{2 \alpha t}\left(\alpha\left(1+\left\|\nabla u_{h}\right\|^{2}\right)+\left(u_{h t},-\Delta_{h} u_{h}\right)\right)\|\nabla \theta\|^{2} .
\end{aligned}
$$


Now multiplying the above inequality by $\tau$ with Young's inequality yields

$$
\begin{aligned}
\tau(t) e^{2 \alpha t}\left\|\theta_{t}\right\|^{2}+\frac{d}{d t} & \left(\tau(t)\left(1+\left\|\nabla u_{h}\right\|^{2}\right) e^{2 \alpha t}\|\nabla \theta\|^{2}\right) \\
& \leq 6 \tau(t) e^{2 \alpha t}\left(\frac{1}{2}\left\|\eta_{t}\right\|^{2}+\left(\left\|\nabla u_{h}\right\|^{2}+\left\|\nabla \widetilde{u}_{h}\right\|^{2}\right)\|\nabla \theta\|^{2}\|\Delta u\|^{2}\right. \\
& \left.+\left(\|\nabla u\|^{2}+\left\|\nabla \widetilde{u}_{h}\right\|^{2}\right)\|\nabla \eta\|^{2}\|\Delta u\|^{2}\right) \\
& +2 e^{2 \alpha t}\left(\left(\tau(t) \alpha+\frac{1}{2}\right)\left(1+\left\|\nabla u_{h}\right\|^{2}\right)+\tau(t)\left\|u_{h t}\right\|\left\|\Delta_{h} u_{h}\right\|\right)\|\nabla \theta\|^{2}
\end{aligned}
$$

An integration of $(3.20)$ with respect to time from 0 to $t$ shows using $\left\|\nabla \widetilde{u}_{h}\right\| \leq\|\nabla u\|($ from Ritz projection) and multiplying the resulting inequality by $e^{-2 \alpha t}$ that

$$
e^{-2 \alpha t} \int_{0}^{t} \tau(s) e^{2 \alpha s}\left\|\theta_{s}\right\|^{2} d s+\tau(t)\left(1+\left\|\nabla u_{h}\right\|^{2}\right)\|\nabla \theta(t)\|^{2}
$$

$$
\begin{aligned}
& \left.\leq C e^{-2 \alpha t} \int_{0}^{t} e^{2 \alpha s}\left(\left\|\eta_{s}\right\|^{2}+\|\nabla u\|^{2}\right)\|\Delta u\|^{2}\|\nabla \eta\|^{2}\right) d s \\
& +C e^{-2 \alpha t} \int_{0}^{t} e^{2 \alpha s}\left(\left(\left\|\nabla u_{h}\right\|^{2}+\|\nabla u\|^{2}\right)\|\Delta u\|^{2}\right. \\
& \left.+(1+\alpha)\left(1+\left\|\nabla u_{h}\right\|^{2}\right)+\left(\tau^{\frac{1}{2}}\left\|u_{h s}\right\|\right)\left(\tau^{\frac{1}{2}}\left\|\Delta_{h} u_{h}\right\|\right)\right)\|\nabla \theta(s)\|^{2} d s .
\end{aligned}
$$

Consequently,

$$
\begin{aligned}
e^{-2 \alpha t} \int_{0}^{t} \tau(s) e^{2 \alpha s}\left\|\theta_{s}\right\|^{2} d s & +\tau(t)\left(1+\left\|\nabla u_{h}\right\|^{2}\right)\|\nabla \theta(t)\|^{2} \\
\leq & \left.C h^{2} e^{-2 \alpha t} \int_{0}^{t} e^{2 \alpha s}\left(\left\|\nabla u_{s}\right\|^{2}+\|\nabla u\|^{2}\right)\|\Delta u\|^{2}\left(\|\Delta u\|^{2}\right)\right) d s \\
& +C e^{-2 \alpha t} \int_{0}^{t} e^{2 \alpha s}\left(\left(\left\|\nabla u_{h}\right\|^{2}+\|\nabla u\|^{2}\right)\|\Delta u\|^{2}\right. \\
& \left.+(1+\alpha)\left(1+\left\|\nabla u_{h}\right\|^{2}\right)+\left(\tau^{\frac{1}{2}}\left\|u_{h s}\right\|\right)\left(\tau^{\frac{1}{2}}\left\|\Delta_{h} u_{h}\right\|\right)\right)\|\nabla \theta(s)\|^{2} d s .
\end{aligned}
$$

From Lemmas 2.2, 2.4 2.5, the first term on right hand side is bounded by

$$
\left.C\left(K_{1}\right) h^{2} e^{-2 \alpha t}\left(\left(1+\| \nabla u_{0}\right) \|^{2}\right)^{2}\left\|u_{0}\right\|_{H^{2}}^{2}+\left\|f_{0}\right\|^{2}+\int_{0}^{t} e^{2 \alpha s}\left\|f_{s}\right\|_{-1}^{2} d s\right) .
$$

For bounding the second term on right hand side, apply the previous theorem 3.3 to obtain a bound as

$$
\left(\left(\left\|\nabla u_{h}\right\|^{2}+\|\nabla u\|^{2}\right)\|\Delta u\|^{2}+(1+\alpha)\left(1+\left\|\nabla u_{h}\right\|^{2}\right)+\left(\tau^{\frac{1}{2}}\left\|u_{h s}\right\|\right)\left(\tau^{\frac{1}{2}}\left\|\Delta_{h} u_{h}\right\|\right)\right) .
$$

By applying Lemma 2.2, 2.5, 3.1, 3.3, 3.4 the above term is bounded. Consequently, the third term in the right hand side is bounded by

$$
\left.C\left(K_{1}, K_{5}\right) h^{2} e^{-2 \alpha t}\left(\left(1+\| \nabla u_{0}\right) \|^{2}\right)^{2}\left\|u_{0}\right\|_{H^{2}}^{2}+\left\|f_{0}\right\|^{2}+\int_{0}^{t} e^{2 \alpha s}\left\|f_{s}\right\|_{-1}^{2} d s\right) .
$$

Altogether, we arrive at

$e^{-2 \alpha t} \int_{0}^{t} \tau(s) e^{2 \alpha s}\left\|\theta_{s}\right\|^{2} d s+\tau(t)\left(1+\left\|\nabla u_{h}\right\|^{2}\right)\|\nabla \theta(t)\|^{2}$

$$
\leq C\left(K_{1}, K_{5}\right) h^{2} e^{-2 \alpha t}\left(\left(1+\left\|\nabla u_{0}\right\|^{2}\right)^{2}\left\|u_{0}\right\|_{H^{2}}^{2}+\left\|f_{0}\right\|^{2}+\int_{0}^{t} e^{2 \alpha s}\left\|f_{s}\right\|_{-1}^{2} d s\right) .
$$

This completes the rest of the proof. 
An application of triangle inequality with the estimate of $\|\nabla \eta\|$ from $(3.9)$ and the estimate $\|\nabla \theta\|$ from Theorem 3.4 yields the following main result of this section.

Theorem 3.5. Let $u_{0} \in H^{2} \cap H_{0}^{1}(\Omega), f \in L^{\infty}\left(L^{2}\right)$ and $f_{t} \in L^{2}\left(H^{-1}\right)$. Then, there holds:

$$
\left\|\left(u-u_{h}\right)(t)\right\|^{2}+\tau(t)\left\|\nabla\left(u-u_{h}\right)(t)\right\|^{2}
$$

$$
\leq C\left(K_{1}, K_{5}\right) h^{2} e^{-2 \alpha t}\left(\left(1+\left\|\nabla u_{0}\right\|^{2}\right)^{2}\left\|u_{0}\right\|_{H^{2}}^{2}+\left\|f_{0}\right\|^{2}+\int_{0}^{t} e^{2 \alpha s}\left\|f_{s}\right\|_{-1}^{2} d s\right) .
$$

Remark 3.1. (i) Note that from the theorem 3.5, the estimates are valid uniformly in time.

(ii) When $f=0$, or $f, f_{t}=O\left(e^{-\gamma_{0} t}\right)$, the following exponential decay property for the error estimates holds:

$$
\left\|\left(u-u_{h}\right)(t)\right\|+\tau(t)\left\|\nabla\left(u-u_{h}\right)(t)\right\| \leq C\left(K_{1}\right) h e^{-2 \gamma t},
$$

where $K_{1}$ depends on $\left\|\nabla u_{0}\right\|^{2}$, and $\gamma=\alpha$, in case $f=0$ and $\gamma=\min \left(\alpha, \gamma_{0}\right)$ for $f=$ $O\left(e^{-\gamma_{0} t}\right)$.

\section{Backward Euler Method}

This section is devoted to a completely discrete scheme which is based on a backward Euler method. Let $\left\{t_{n}\right\}_{n=0}^{N}$ be a uniform partition of $[0, T]$, and $t_{n}=n k$, with time step $k>0$. For smooth function $\phi$ defined on $[0, T]$, set $\phi^{n}=\phi\left(t_{n}\right)$ and $\bar{\partial}_{t} \phi^{n}=\frac{\left(\phi^{n}-\phi^{n-1}\right)}{k}$.

Now the backward Euler method applied to (3.1) determines a sequence of functions $\left\{U^{n}\right\}_{n \geq 1} \in V_{h}$ as solution of

$$
\begin{aligned}
& \left(\bar{\partial}_{t} U^{n}, \varphi_{h}\right)+\left(1+\left\|\nabla U^{n}\right\|^{2}\right)\left(\nabla U^{n}, \nabla \varphi_{h}\right)=\left(f^{n}, \varphi_{h}\right) \quad \forall \varphi_{h} \in V_{h}, \\
& U^{0}=u_{0 h} .
\end{aligned}
$$

Now we derive a priori bounds for the solution $\left\{U^{n}\right\}_{n \geq 1}$.

Lemma 4.1. The discrete solution $U^{N}, N \geq 1$ of 4.1 satisfies

$$
\left\|U^{N}\right\| \leq\left\|U^{0}\right\|+2 k \sum_{n=1}^{N}\left\|f^{n}\right\|
$$

Proof. Set $\varphi_{h}=U^{n}$ in 4.1 and obtain

$$
\left(\bar{\partial}_{t} U^{n}, U^{n}\right)+\left(1+\left\|\nabla U^{n}\right\|^{2}\right)\left\|\nabla U^{n}\right\|^{2}=\left(f^{n}, U^{n}\right) .
$$

Note that

$$
\left(\bar{\partial}_{t} U^{n}, U^{n}\right)=\frac{1}{2} \bar{\partial}_{t}\left\|U^{n}\right\|^{2}+\frac{k}{2}\left\|\bar{\partial}_{t} U^{n}\right\|^{2}
$$

Therefore,

$$
\begin{aligned}
\frac{1}{2} \bar{\partial}_{t}\left\|U^{n}\right\|^{2}+\frac{k}{2}\left\|\bar{\partial}_{t} U^{n}\right\|^{2}+\left(1+\left\|\nabla U^{n}\right\|^{2}\right)\left\|\nabla U^{n}\right\|^{2} & =\left(f^{n}, U^{n}\right) \\
& \leq\left\|f^{n}\right\|\left\|U^{n}\right\|
\end{aligned}
$$

Consequently,

$$
\frac{1}{2} \bar{\partial}_{t}\left\|U^{n}\right\|^{2} \leq\left\|f^{n}\right\|\left\|U^{n}\right\| .
$$

That is,

$$
\left(\left\|U^{n}\right\|^{2}-\left\|U^{n-1}\right\|\right) \leq 2 k\left\|f^{n}\right\|\left\|U^{n}\right\| .
$$


Sum it up from $n=1$ to $N$ to obtain

$$
\left\|U^{N}\right\|^{2} \leq\left\|U^{0}\right\|^{2}+2 k \sum_{n=1}^{N}\left\|f^{n}\right\|\left\|U^{n}\right\| .
$$

Let $N^{*} \in\{0, \cdots, N\}$ such that $U^{N^{*}}=\max _{0 \leq n \leq N}\left\|U^{n}\right\|$.

Since (4.4) is true for $N=N^{*}$, therefore,

$$
\begin{aligned}
\left\|U^{N^{*}}\right\|^{2} & \leq\left\|U^{0}\right\|^{2}+2 k \sum_{n=1}^{N}\left\|f^{n}\right\|\left\|U^{n}\right\| \\
& \leq\left(\left\|U^{0}\right\|+2 k \sum_{n=1}^{N}\left\|f^{n}\right\|\right)\left\|U^{N^{*}}\right\| .
\end{aligned}
$$

Consequently,

$$
\left\|U^{N}\right\| \leq\left\|U^{N^{*}}\right\| \leq\left(\left\|U^{0}\right\|+2 k \sum_{n=1}^{N}\left\|f^{n}\right\|\right) .
$$

This completes the rest of the proof.

Remark 4.1. Set $\varphi_{h}=-\Delta_{h} U^{n}$ in 4.1 and obtain

$$
\left(\bar{\partial}_{t} U^{n},-\Delta_{h} U^{n}\right)+\left(1+\left\|\nabla U^{n}\right\|^{2}\right)\left\|\Delta_{h} U^{n}\right\|^{2}=\left(f^{n},-\Delta_{h} U^{n}\right) .
$$

Note that

$$
\left(\bar{\partial}_{t} U^{n},-\Delta_{h} U^{n}\right)=\left(\bar{\partial}_{t} \nabla U^{n}, \nabla U^{n}\right)=\frac{1}{2} \bar{\partial}_{t}\left\|\nabla U^{n}\right\|^{2}+\frac{k}{2}\left\|\bar{\partial}_{t} \nabla U^{n}\right\|^{2}
$$

Therefore,

$$
\begin{aligned}
\frac{1}{2} \bar{\partial}_{t}\left\|\nabla U^{n}\right\|^{2}+\frac{k}{2}\left\|\bar{\partial}_{t} \nabla U^{n}\right\|^{2}+ & \left(1+\left\|\nabla U^{n}\right\|^{2}\right)\left\|\Delta_{h} U^{n}\right\|^{2} \\
& =\left(f^{n},-\Delta_{h} U^{n}\right) \\
& \leq \frac{1}{2}\left\|f^{n}\right\|^{2}+\frac{1}{2}\left\|\Delta_{h} U^{n}\right\|^{2} \leq \frac{1}{2}\left\|f^{n}\right\|^{2}+\frac{1}{2}\left(1+\left\|\nabla U^{n}\right\|^{2}\right)\left\|\Delta_{h} U^{n}\right\|^{2}
\end{aligned}
$$

Consequently,

$$
\bar{\partial}_{t}\left\|\nabla U^{n}\right\|^{2}+\left(1+\left\|\nabla U^{n}\right\|^{2}\right)\left\|\Delta_{h} U^{n}\right\|^{2} \leq\left\|f^{n}\right\|^{2},
$$

and hence $\left\|\nabla U^{n}\right\|^{2}$ is bounded.

\subsection{Existence and uniqueness of discrete solution}

Theorem 4.1. (Brouwer's fixed point theorem) [7]. Let $H$ be a finite dimensional Hilbert space with inner product (...) and $\|$.$\| . Let g: H \rightarrow H$ be a continuous function. If there exist $R>0$ such that $(g(z), z)>0 \forall z$ with $\|z\|=R$, then there exists $z^{*} \in H$ such that $\|z\| \leq R$ and $g\left(z^{*}\right)=0$.

Theorem 4.2. Given $U^{n-1}$, the discrete problem (4.1) has a unique solution $U^{n}, n \geq 1$.

Proof. Given $U^{n-1}$, define a function $\mathbb{F}: V_{h} \rightarrow V_{h}$ for a fixed $n$ by

$$
\left(\mathbb{F}(v), \varphi_{h}\right)=\left(v, \varphi_{h}\right)+k\left(1+\|\nabla v\|^{2}\right)\left(\nabla v, \nabla \varphi_{h}\right)-k\left(f^{n}, \varphi_{h}\right)-\left(U^{n-1}, \varphi_{h}\right)
$$


Define a norm on $V_{h}$ as

$$
\|\mid v\| \|=\left(\|v\|^{2}+k\|\nabla v\|^{2}\right)^{\frac{1}{2}}
$$

then $\mathbb{F}$ is continuous by sequential criterion. Now substituting $\varphi_{h}=v$ in 4.7 to obtain

$$
\begin{aligned}
(\mathbb{F}(v), v) & =\|v\|^{2}+k\left(1+\|\nabla v\|^{2}\right)\|\nabla v\|^{2}-k\left(f^{n}, v\right)-\left(U^{n-1}, v\right) \\
& \geq\|v\|^{2}+k\|\nabla v\|^{2}-k\left(\left\|f^{n}\right\|+\left\|U^{n-1}\right\|\right)\|v\|
\end{aligned}
$$

Choosing $R$ in such a way that $\mid\|v\| \|=R$ with $R-k\left(\left\|f^{n}\right\|+\left\|U^{n-1}\right\|\right)>0$ and hence,

$$
(\mathbb{F}(v), v) \geq R\left(R-k\left(\left\|f^{n}\right\|+\left\|U^{n-1}\right\|\right)\right)>0
$$

A use of theorem 4.1 would provide us the existence of $\left\{U^{n}\right\}_{n>1}$.

Now to prove uniqueness, set $W^{n}=U_{1}^{n}-U_{2}^{n}$, where $U_{1}^{n}$ and $U_{2}^{n}$ are the solutions of (4.1). Then, $W^{n}$ satisfy

$$
\left(\bar{\partial}_{t} W^{n}, \varphi_{h}\right)+\left(\left(1+\left\|\nabla U_{1}^{n}\right\|\right)^{2} \nabla U_{1}^{n}-\left(1+\left\|\nabla U_{2}^{n}\right\|\right)^{2} \nabla U_{2}^{n}, \nabla \varphi_{h}\right)=0
$$

Substitute $\varphi_{h}=W^{n}=U_{1}^{n}-U_{2}^{n}$, we obtain

$$
\left(\bar{\partial}_{t} W^{n}, W^{n}\right)+\left(\left(1+\left\|\nabla U_{1}^{n}\right\|\right)^{2} \nabla U_{1}^{n}-\left(1+\left\|\nabla U_{2}^{n}\right\|\right)^{2} \nabla U_{2}^{n}, \nabla\left(U_{1}^{n}-U_{2}^{n}\right)\right)=0
$$

Using monotonicity property in Lemma 2.8 we observe

$$
\left(\left(1+\left\|\nabla U_{1}^{n}\right\|\right)^{2} \nabla U_{1}^{n}-\left(1+\left\|\nabla U_{2}^{n}\right\|\right)^{2} \nabla U_{2}^{n}, \nabla\left(U_{1}^{n}-U_{2}^{n}\right)\right) \geq\left\|\nabla W^{n}\right\|^{2} \geq 0 .
$$

Consequently

$$
\left(\bar{\partial}_{t} W^{n}, W^{n}\right) \leq 0
$$

and hence

$$
\frac{1}{2 k}\left(\left\|W^{n}\right\|^{2}-\left\|W^{n-1}\right\|\right) \leq 0 .
$$

Taking summation from $n=1$ to $N$ to obtain

$$
\left\|W^{N}\right\|^{2} \leq\left\|W^{0}\right\|^{2} .
$$

Since $W^{0}=0$, it follows that $W^{N}=0$ which leads to a contradiction. Hence, the solution is unique. This completes the rest of the proof.

\subsection{Error Analysis for Backward Euler Method}

In this subsection, we discuss error estimates for fully discrete finite element method.

Now spllit the error $e^{n}=u\left(t_{n}\right)-U^{n}=\left(u\left(t_{n}\right)-\widetilde{u}\left(t_{n}\right)\right)-\left(U^{n}-\widetilde{u}\left(t_{n}\right)\right)=\eta^{n}-\theta^{n}$, where $U^{n}$ is the solution of (4.1) and $u\left(t_{n}\right)$ is the soution of 2.1), and $\eta^{n}=\eta\left(t_{n}\right)$ is defined in (3.8).

Using (2.1) at $t=t_{n}$ and (4.1), the equation in $\theta^{n}$ becomes for all $\phi_{h} \in V_{h}$

$$
\begin{aligned}
\left(\bar{\partial}_{t} \theta^{n}, \varphi_{h}\right)+\left(\left(1+\left\|\nabla U^{n}\right\|^{2}\right) \nabla U^{n}-\right. & \left.\left(1+\left\|\nabla \widetilde{u}\left(t_{n}\right)\right\|^{2}\right) \nabla \widetilde{u}\left(t_{n}\right), \nabla \varphi_{h}\right) \\
= & \left(\bar{\partial}_{t} \eta^{n}, \varphi_{h}\right)+\left(u_{t}\left(t_{n}\right)-\bar{\partial}_{t} u\left(t_{n}\right), \varphi_{h}\right) \\
& +\left(\left(1+\left\|\nabla u\left(t_{n}\right)\right\|^{2}\right) \nabla u\left(t_{n}\right)-\left(1+\left\|\nabla \widetilde{u}\left(t_{n}\right)\right\|^{2}\right) \nabla \widetilde{u}\left(t_{n}\right), \nabla \varphi_{h}\right) .
\end{aligned}
$$

Theorem 4.3. Let $0<\alpha<\frac{\lambda_{1}}{2}$. Choose $k_{0}>0$ such that for $0<k \leq k_{0}$

$$
\left(1+\frac{\lambda_{1} k}{2}\right)>e^{\alpha k}
$$


where $\beta=\left(e^{-\alpha k}-\frac{2}{k \lambda_{1}}\left(1-e^{-\alpha k}\right)\right)>0$ holds. Then, there exists a positive constant $C=C\left(\lambda_{1}, K_{1}\right)$ independent of $h$ and $k$ such that

$$
\begin{aligned}
\left\|\theta^{N}\right\|^{2}+ & k \beta e^{-2 \alpha t_{N}} \sum_{n=1}^{N} e^{2 \alpha t_{n}}\left\|\nabla \theta^{n}\right\|^{2} \\
& \leq C\left(\lambda_{1}, K_{1}\right) e^{-2 \alpha t_{N}}\left(k^{2}+h^{2}\right)\left(\left\|u_{0}\right\|_{H^{3}}^{2}+\left(\left(1+\left\|\nabla u_{0}\right\|^{2}\right)^{2}\left\|u_{0}\right\|_{H^{2}}^{2}+\left\|f_{0}\right\|^{2}\right)\right. \\
& \left.+\int_{0}^{t_{N}} e^{2 \alpha s}\left\|f_{s}\right\|_{-1}^{2} d s\right) .
\end{aligned}
$$

Proof. Multiplying 4.12) by $e^{\alpha t_{n}}$ and putting $\varphi_{h}=e^{\alpha t_{n}} \theta^{n}=\widehat{\theta}^{n}$, we obtain

$$
\begin{aligned}
\left(e^{\alpha t_{n}} \bar{\partial}_{t} \theta^{n}, \widehat{\theta}^{n}\right)+e^{\alpha t_{n}} & \left(\left(1+\left\|\nabla U^{n}\right\|^{2}\right) \nabla U^{n}-\left(1+\left\|\nabla \widetilde{u}\left(t_{n}\right)\right\|^{2}\right) \nabla \widetilde{u}\left(t_{n}\right), \nabla \widehat{\theta}^{n}\right) \\
& =e^{\alpha t_{n}}\left(\bar{\partial}_{t} \eta^{n}, \widehat{\theta}^{n}\right)+e^{\alpha t_{n}}\left(u_{t}\left(t_{n}\right)-\bar{\partial}_{t} u\left(t_{n}\right), \widehat{\theta}^{n}\right) \\
& +e^{\alpha t_{n}}\left(\left(1+\left\|\nabla u\left(t_{n}\right)\right\|^{2}\right) \nabla u\left(t_{n}\right)-\left(1+\left\|\nabla \widetilde{u}\left(t_{n}\right)\right\|^{2}\right) \nabla \widetilde{u}\left(t_{n}\right), \nabla \widehat{\theta}^{n}\right) .
\end{aligned}
$$

Note that

$$
e^{\alpha t_{n}} \bar{\partial}_{t} \theta^{n}=e^{\alpha k} \bar{\partial}_{t} \widehat{\theta}^{n}-\frac{\left(e^{\alpha k}-1\right)}{k} \widehat{\theta}^{n}
$$

Now by monotonicity property given in Lemma 2.8

$$
e^{2 \alpha t_{n}}\left(\left(1+\left\|\nabla U^{n}\right\|^{2}\right) \nabla U^{n}-\left(1+\left\|\nabla \widetilde{u}\left(t_{n}\right)\right\|^{2}\right) \nabla \widetilde{u}\left(t_{n}\right), \nabla\left(U^{n}-\widetilde{u}\left(t_{n}\right)\right)\right) \geq\left\|\nabla \widehat{\theta}^{n}\right\|^{2} .
$$

Therefore, using 4.16-4.17 and Ritz-projection, we obtain from 4.15

$$
\begin{aligned}
e^{\alpha k}\left(\bar{\partial}_{t} \widehat{\theta}^{n}, \widehat{\theta}^{n}\right)- & \frac{\left(e^{\alpha k}-1\right)}{k}\left\|\widehat{\theta}^{n}\right\|^{2}+\left\|\nabla \widehat{\theta}^{n}\right\|^{2} \\
& \leq e^{\alpha t_{n}}\left(\bar{\partial}_{t} \eta^{n}, \widehat{\theta}^{n}\right)+e^{\alpha t_{n}}\left(u_{t}\left(t_{n}\right)-\bar{\partial}_{t} u\left(t_{n}\right), \widehat{\theta}^{n}\right) \\
& +e^{\alpha t_{n}}\left(\left(\left\|\nabla u\left(t_{n}\right)\right\|^{2}-\left\|\nabla \widetilde{u}\left(t_{n}\right)\right\|^{2}\right) \nabla u\left(t_{n}\right), \nabla \widehat{\theta}^{n}\right) .
\end{aligned}
$$

Note that

$$
\left(\overline{\partial_{t}} \widehat{\theta}^{n}, \widehat{\theta}^{n}\right)=\frac{1}{2} \bar{\partial}_{t}\left\|\widehat{\theta}^{n}\right\|^{2}+\frac{k}{2}\left\|\bar{\partial}_{t} \widehat{\theta}^{n}\right\|^{2} \geq \frac{1}{2} \bar{\partial}_{t}\left\|\widehat{\theta}^{n}\right\|^{2}
$$

and

$$
\left\|\widehat{\theta}^{n}\right\|^{2} \leq \frac{1}{\lambda_{1}}\left\|\nabla \widehat{\theta}^{n}\right\|^{2}
$$

Now, using Poincare's inequality and Young's inequality, we estimate the first, second and third terms in the right hand side of (4.18) as follows

$$
\begin{gathered}
e^{\alpha t_{n}}\left(\bar{\partial}_{t} \eta^{n}, \widehat{\theta}^{n}\right) \leq \frac{3 e^{2 \alpha t_{n}}}{2 \lambda_{1}}\left\|\bar{\partial}_{t} \eta^{n}\right\|^{2}+\frac{1}{6}\left\|\nabla \widehat{\theta}^{n}\right\|^{2}, \\
e^{\alpha t_{n}}\left(u_{t}\left(t_{n}\right)-\bar{\partial}_{t} u\left(t_{n}\right), \widehat{\theta}^{n}\right) \leq \frac{3}{2 \lambda_{1}} e^{2 \alpha t_{n}}\left\|u_{t}\left(t_{n}\right)-\bar{\partial}_{t} u\left(t_{n}\right)\right\|^{2}+\frac{1}{6}\left\|\nabla \widehat{\theta}^{n}\right\|^{2},
\end{gathered}
$$

and

$$
e^{\alpha t_{n}}\left(\left(\left\|\nabla u\left(t_{n}\right)\right\|^{2}-\left\|\nabla \widetilde{u}\left(t_{n}\right)\right\|^{2}\right) \nabla u\left(t_{n}\right), \nabla \widehat{\theta}^{n}\right) \leq 6 e^{2 \alpha t_{n}}\left\|\nabla u\left(t_{n}\right)\right\|^{4}\left\|\nabla \eta^{n}\right\|^{2}+\frac{1}{6}\left\|\nabla \widehat{\theta}^{n}\right\|^{2}
$$


Therefore, using 4.19- -4.23 in 4.18 and multiplying by $2 e^{-\alpha k}$ the resulting inequality, we arrive at

$$
\begin{aligned}
\bar{\partial}_{t}\left\|\widehat{\theta}^{n}\right\|^{2}+\left(e^{-\alpha k}-\frac{2}{k \lambda_{1}}\left(1-e^{-\alpha k}\right)\right)\left\|\nabla \widehat{\theta}^{n}\right\|^{2} & \leq \frac{3}{2 \lambda_{1}} e^{-\alpha k} e^{2 \alpha t_{n}}\left(\left\|\bar{\partial}_{t} \eta^{n}\right\|^{2}+\left\|u_{t}\left(t_{n}\right)-\bar{\partial}_{t} u\left(t_{n}\right)\right\|^{2}\right) \\
& +6 e^{-\alpha k} e^{2 \alpha t_{n}}\left\|\nabla u\left(t_{n}\right)\right\|^{4}\left\|\nabla \eta^{n}\right\|^{2} .
\end{aligned}
$$

With $0<\alpha<\frac{\lambda_{1}}{2}$, choose $k_{0}>0$ such that for $0<k \leq k_{0}, 4.13$ is satisfied. Then $\beta=$ $\left(e^{-\alpha k}-\frac{2}{k \lambda_{1}}\left(1-e^{-\alpha k}\right)\right)>0$. Therefore, multiplying 4.24 by $k$, and summing over $n=1$ to $N$, we arrive at

$$
\begin{aligned}
\left\|\widehat{\theta}^{N}\right\|^{2}+k \beta \sum_{n=1}^{N}\left\|\nabla \widehat{\theta}^{n}\right\|^{2} \leq\left\|\widehat{\theta}^{0}\right\|^{2} & +\frac{3}{2 \lambda_{1}} k e^{-\alpha k} \sum_{n=1}^{N} e^{2 \alpha t_{n}}\left(\left\|\bar{\partial}_{t} \eta^{n}\right\|^{2}+\left\|u_{t}\left(t_{n}\right)-\bar{\partial}_{t} u\left(t_{n}\right)\right\|^{2}\right) \\
& +6 k e^{-\alpha k} \sum_{n=1}^{N} e^{2 \alpha t_{n}}\left\|\nabla u\left(t_{n}\right)\right\|^{4}\left\|\nabla \eta^{n}\right\|^{2} .
\end{aligned}
$$

Note that

$$
\begin{aligned}
\left\|\bar{\partial}_{t} \eta^{n}\right\|^{2}= & \frac{1}{k^{2}}\left(\int_{t_{n-1}}^{t_{n}}(u-\widetilde{u})_{t} d s\right)^{2} \\
& \leq \frac{1}{k} \int_{t_{n-1}}^{t_{n}}\left\|\eta_{t}\right\|^{2} d s \leq \frac{1}{k} C h^{2} \int_{t_{n-1}}^{t_{n}}\left\|\nabla u_{t}(s)\right\|^{2} d s .
\end{aligned}
$$

Therefore, the second term on the right hand side of 4.25 can be bounded by

$$
\begin{aligned}
\frac{3}{2 \lambda_{1}} k e^{-\alpha k} \sum_{n=1}^{N} e^{2 \alpha t_{n}}\left\|\bar{\partial}_{t} \eta^{n}\right\|^{2} & \leq C\left(\lambda_{1}\right) h^{2} e^{-\alpha k} \sum_{n=1}^{N} \int_{t_{n-1}}^{t_{n}} e^{2 \alpha t_{n}}\left\|\nabla u_{t}(s)\right\|^{2} d s \\
& =C\left(\lambda_{1}\right) h^{2} e^{-\alpha k} e^{2 \alpha k} \sum_{n=1}^{N} \int_{t_{n-1}}^{t_{n}} e^{2 \alpha t_{n-1}}\left\|\nabla u_{t}(s)\right\|^{2} d s \\
& \leq C\left(\lambda_{1}\right) h^{2} \int_{0}^{t_{N}} e^{2 \alpha s}\left\|\nabla u_{t}(s)\right\|^{2} d s .
\end{aligned}
$$

By the Taylor series expansion of $u$ around $t_{n}$ in the interval $\left(t_{n-1}, t_{n}\right)$, we obtain

$$
\begin{aligned}
\left\|u_{t}\left(t_{n}\right)-\bar{\partial}_{t} u\left(t_{n}\right)\right\|^{2} & \leq \frac{1}{k^{2}}\left(\int_{t_{n-1}}^{t_{n}}\left(t_{n}-s\right)\left\|u_{t t}(s)\right\| d s\right)^{2} \\
& \leq \frac{1}{k^{2}}\left(\int_{t_{n-1}}^{t_{n}}\left(t_{n}-s\right)^{2} d s\right)\left(\int_{t_{n-1}}^{t_{n}}\left\|u_{t t}(s)\right\|^{2} d s\right) \\
& =\frac{k}{3} \int_{t_{n-1}}^{t_{n}}\left\|u_{t t}(s)\right\|^{2} d s,
\end{aligned}
$$

and the third term on the right hand of 4.25 side is now bounded by

$$
\begin{aligned}
\frac{3}{2 \lambda_{1}} k \sum_{n=1}^{N} e^{2 \alpha t_{n}}\left\|u_{t}\left(t_{n}\right)-\bar{\partial}_{t} u\left(t_{n}\right)\right\|^{2} & \leq \frac{1}{2 \lambda_{1}} k^{2} e^{-\alpha k} \sum_{n=1}^{N} \int_{t_{n-1}}^{t_{n}} e^{2 \alpha t_{n}}\left\|u_{t t}(s)\right\|^{2} d s \\
& \leq \frac{1}{2 \lambda_{1}} k^{2} e^{-\alpha k} e^{2 \alpha k} \sum_{n=1}^{N} \int_{t_{n-1}}^{t_{n}} e^{2 \alpha s}\left\|u_{t t}(s)\right\|^{2} d s \\
& \leq C\left(\lambda_{1}\right) k^{2} \int_{0}^{t_{N}} e^{2 \alpha s}\left\|u_{t t}(s)\right\|^{2} d s .
\end{aligned}
$$


For the last term on the right hand side of 4.25 bound is

$$
\begin{aligned}
6 k e^{-\alpha k} \sum_{n=1}^{N} e^{2 \alpha t_{n}}\left\|\nabla u\left(t_{n}\right)\right\|^{4}\left\|\nabla \eta^{n}\right\|^{2} & \leq C h^{2} e^{-\alpha k} k \sum_{n=1}^{N} e^{2 \alpha t_{n}}\left\|\nabla u\left(t_{n}\right)\right\|^{4}\left\|u\left(t_{n}\right)\right\|_{H^{2}}^{2} \\
& \leq C h^{2} e^{-\alpha k} K_{1}\left(k \sum_{n=1}^{N}\left\|\widehat{u}\left(t_{n}\right)\right\|_{H^{2}}^{2}\right) .
\end{aligned}
$$

Therefore, from 4.25 we arrive at

$$
\begin{aligned}
\left\|\widehat{\theta}^{N}\right\|^{2}+k \beta \sum_{n=1}^{N}\left\|\nabla \widehat{\theta}^{n}\right\|^{2} & \leq\left\|\theta^{0}\right\|^{2}+C\left(\lambda_{1}\right) h^{2} \int_{0}^{t_{N}} e^{2 \alpha s}\left\|\nabla u_{t}(s)\right\|^{2} d s+C\left(\lambda_{1}\right) k^{2} \int_{0}^{t_{N}} e^{2 \alpha s}\left\|u_{t t}(s)\right\|^{2} d s \\
& +C\left(K_{1}\right) h^{2} e^{-\alpha k}\left\|u\left(t_{n}\right)\right\|_{H^{2}}^{2}\left(k \sum_{n=1}^{N} e^{2 \alpha t_{n}}\right) .
\end{aligned}
$$

With a choice $U^{0}=\widetilde{u}(0), \theta^{0}=0$. But with $U^{0}=P_{h} u_{0}$,

$$
\left\|\theta^{0}\right\| \leq C h\left\|\nabla u_{0}\right\|
$$

Multiply (4.31) by $e^{-2 \alpha t_{N}}$ to obtain

$$
\begin{aligned}
\left\|\theta^{N}\right\|^{2}+k \beta e^{-2 \alpha t_{N}} \sum_{n=1}^{N} e^{2 \alpha t_{n}}\left\|\nabla \theta^{n}\right\|^{2} & \leq e^{-2 \alpha t_{N}}\left\|\theta^{0}\right\|^{2}+C\left(\lambda_{1}\right) e^{-2 \alpha t_{N}} h^{2} \int_{0}^{t_{N}} e^{2 \alpha s}\left\|\nabla u_{t}(s)\right\|^{2} d s \\
& +C\left(\lambda_{1}\right) e^{-2 \alpha t_{N}} k^{2} \int_{0}^{t_{N}} e^{2 \alpha s}\left\|u_{t t}(s)\right\|^{2} d s \\
& +C\left(K_{1}\right) e^{-\alpha k}\left(e^{-2 \alpha t_{N}} k \sum_{n=1}^{N} e^{2 \alpha t_{n}}\right)\left\|u\left(t_{n}\right)\right\|_{H^{2}}^{2} h^{2} .
\end{aligned}
$$

Note that

$$
e^{-2 \alpha t_{N}}\left(k \sum_{n=1}^{N} e^{2 \alpha t_{n}}\right)=e^{-2 \alpha t_{N}} \frac{1}{e^{2 \alpha k}-1} k e^{2 \alpha k}\left(e^{2 \alpha t_{N}}-1\right) \leq C .
$$

By using Lemma 2.4 and 2.7 the second and third term in the right hand side of 4.32 are bounded respectively.

Therefore

$$
\begin{aligned}
\left\|\theta^{N}\right\|^{2}+ & k \beta e^{-2 \alpha t_{N}} \sum_{n=1}^{N} e^{2 \alpha t_{n}}\left\|\nabla \theta^{n}\right\|^{2} \\
& \leq C\left(\lambda_{1}, K_{1}\right) e^{-2 \alpha t_{N}}\left(k^{2}+h^{2}\right)\left(\left\|u_{0}\right\|_{H^{3}}^{2}+\left(\left(1+\left\|\nabla u_{0}\right\|^{2}\right)^{2}\left\|u_{0}\right\|_{H^{2}}^{2}+\left\|f_{0}\right\|^{2}\right)\right. \\
& \left.+\int_{0}^{t_{N}} e^{2 \alpha s}\left\|f_{s}\right\|_{-1}^{2} d s\right) .
\end{aligned}
$$

This completes the rest of the proof.

Since from 3.9$]\left\|\nabla \eta^{n}\right\| \leq C h\left\|u\left(t_{n}\right)\right\|_{H^{2}}$, is known, to in order to, estimate of $\left\|\nabla u\left(t_{n}\right)-U^{n}\right\|$, it is enough to estimate $\left\|\nabla \theta^{n}\right\|$. 
Theorem 4.4. Assume that $0<\alpha<\frac{\lambda_{1}}{2}$ and choose $k_{0}>0$ be such that for $0<k \leq k_{0}$, 4.13 is true. Then, there exists a positive constant $C=C(\alpha, K)$ such that

$$
\begin{aligned}
k e^{-2 \alpha t_{N}} \sum_{n=1}^{N} & \left\|\bar{\partial}_{t} \widehat{\theta}^{n}\right\|^{2}+e^{-\alpha k}\left(1+\left\|\nabla U^{N}\right\|^{2}\right)\left\|\nabla \theta^{N}\right\|^{2} \\
& \leq C(\alpha, K) e^{-2 \alpha t_{N}}\left(h^{2}+k^{2}\right)\left(\left\|u_{0}\right\|_{H^{3}}^{2}+\left(\left(1+\left\|\nabla u_{0}\right\|^{2}\right)^{2}\left\|u_{0}\right\|_{H^{2}}^{2}+\left\|f_{0}\right\|^{2}\right)\right. \\
& \left.+\int_{0}^{t_{N}} e^{2 \alpha s}\left\|f_{s}\right\|_{-1}^{2} d s\right),
\end{aligned}
$$

where $\beta=\left(e^{-\alpha k}-\frac{2}{k \lambda_{1}}\left(1-e^{-\alpha k}\right)\right)>0$, and $K$ depends on $\nabla u_{0}$.

Proof. Multiply the equation 4.12 by $e^{\alpha t_{n}}$ and then putting $\varphi_{h}=\bar{\partial}_{t} \widehat{\theta}^{n}$, we obtain

$$
\begin{aligned}
\left(e^{\alpha t_{n}} \bar{\partial}_{t} \theta^{n}, \bar{\partial}_{t} \widehat{\theta}^{n}\right)+e^{\alpha t_{n}} & \left(\left(1+\left\|\nabla U^{n}\right\|^{2}\right) \nabla U^{n}-\left(1+\left\|\nabla \widetilde{u}\left(t_{n}\right)\right\|^{2}\right) \nabla \widetilde{u}\left(t_{n}\right), \nabla \bar{\partial}_{t} \widehat{\theta}^{n}\right) \\
& =e^{\alpha t_{n}}\left(\bar{\partial}_{t} \eta^{n}, \bar{\partial}_{t} \widehat{\theta}^{n}\right)+e^{\alpha t_{n}}\left(u_{t}\left(t_{n}\right)-\bar{\partial}_{t} u\left(t_{n}\right), \bar{\partial}_{t} \widehat{\theta}^{n}\right) \\
& +e^{2 \alpha t_{n}}\left(\left(1+\left\|\nabla u\left(t_{n}\right)\right\|^{2}\right) \nabla u\left(t_{n}\right)-\left(1+\left\|\nabla \widetilde{u}\left(t_{n}\right)\right\|^{2}\right) \nabla \widetilde{u}\left(t_{n}\right), \nabla \bar{\partial}_{t} \widehat{\theta}^{n}\right) .
\end{aligned}
$$

Using (4.16) in 4.35), we find that

$$
\begin{aligned}
e^{\alpha k}\left\|\bar{\partial}_{t} \widehat{\theta}^{n}\right\|^{2}+e^{\alpha t_{n}} & \left(\left(1+\left\|\nabla U^{n}\right\|^{2}\right) \nabla U^{n}-\left(1+\left\|\nabla \widetilde{u}\left(t_{n}\right)\right\|^{2}\right) \nabla \widetilde{u}\left(t_{n}\right), \nabla \bar{\partial}_{t} \widehat{\theta}^{n}\right) \\
& =\frac{\left(e^{\alpha k}-1\right)}{k}\left(\widehat{\theta}^{n}, \bar{\partial}_{t} \widehat{\theta}^{n}\right)+e^{\alpha t_{n}}\left(\bar{\partial}_{t} \eta^{n}, \bar{\partial}_{t} \widehat{\theta}^{n}\right)+e^{\alpha t_{n}}\left(u_{t}\left(t_{n}\right)-\bar{\partial}_{t} u\left(t_{n}\right), \bar{\partial}_{t} \widehat{\theta}^{n}\right) \\
& +e^{2 \alpha t_{n}}\left(\left(1+\left\|\nabla u\left(t_{n}\right)\right\|^{2}\right) \nabla u\left(t_{n}\right)-\left(1+\left\|\nabla \widetilde{u}\left(t_{n}\right)\right\|^{2}\right) \nabla \widetilde{u}\left(t_{n}\right), \nabla \bar{\partial}_{t} \widehat{\theta}^{n}\right) .
\end{aligned}
$$

For the second term on the left hand side of (4.36), use Ritz projection to rewrite it as

$$
\begin{aligned}
e^{\alpha t_{n}}\left(\left(1+\left\|\nabla U^{n}\right\|^{2}\right) \nabla U^{n}-\right. & \left.\left(1+\left\|\nabla \widetilde{u}\left(t_{n}\right)\right\|^{2}\right) \nabla \widetilde{u}\left(t_{n}\right), \nabla \bar{\partial}_{t} \widehat{\theta}^{n}\right) \\
& =\left(\nabla \widehat{\theta}^{n}, \nabla \bar{\partial}_{t} \widehat{\theta}^{n}\right)+\left\|\nabla U^{n}\right\|^{2}\left(\nabla \widehat{\theta}^{n}, \nabla \bar{\partial}_{t} \widehat{\theta}^{n}\right) \\
& -e^{\alpha t_{n}}\left(\left(\left\|\nabla U^{n}\right\|^{2}-\left\|\nabla \widetilde{u}\left(t_{n}\right)\right\|^{2}\right) \Delta u\left(t_{n}\right), \bar{\partial}_{t} \widehat{\theta}^{n}\right) .
\end{aligned}
$$

Note that

$$
\left(\nabla \widehat{\theta}^{n}, \nabla \bar{\partial}_{t} \widehat{\theta}^{n}\right)=\left(\nabla \widehat{\theta}^{n}, \frac{\nabla \widehat{\theta}^{n}-\nabla \widehat{\theta}^{n-1}}{k}\right) \geq \frac{1}{2} \bar{\partial}_{t}\left\|\nabla \widehat{\theta}^{n}\right\|^{2}
$$

The fourth term on the right hand side of 4.36), can be bounded using Ritz projection as

$$
\begin{aligned}
e^{\alpha t_{n}}\left(\left(1+\left\|\nabla u\left(t_{n}\right)\right\|^{2}\right) \nabla u\left(t_{n}\right)-\right. & \left.\left(1+\left\|\nabla \widetilde{u}\left(t_{n}\right)\right\|^{2}\right) \nabla \widetilde{u}\left(t_{n}\right), \nabla \bar{\partial}_{t} \widehat{\theta}^{n}\right) \\
& =e^{\alpha t_{n}}\left(\left\|\nabla u\left(t_{n}\right)\right\|^{2} \nabla u\left(t_{n}\right)-\left\|\nabla \widetilde{u}\left(t_{n}\right)\right\|^{2} \nabla \widetilde{u}\left(t_{n}\right), \nabla \bar{\partial}_{t} \widehat{\theta}^{n}\right) \\
& =e^{\alpha t_{n}}\left(\left(\left\|\nabla u\left(t_{n}\right)\right\|^{2}-\left\|\nabla \widetilde{u}\left(t_{n}\right)\right\|^{2}\right) \nabla \widetilde{u}\left(t_{n}\right), \nabla \bar{\partial}_{t} \widehat{\theta}^{n}\right) \\
& =-e^{\alpha t_{n}}\left(\left(\left\|\nabla u\left(t_{n}\right)\right\|^{2}-\left\|\nabla \widetilde{u}\left(t_{n}\right)\right\|^{2}\right) \Delta u\left(t_{n}\right), \bar{\partial}_{t} \widehat{\theta}^{n}\right) \\
& \leq C e^{\alpha t_{n}}\left\|\nabla u\left(t_{n}\right)\right\|\left\|\nabla \eta^{n}\right\| \Delta u\left(t_{n}\right)\left\|\bar{\partial}_{t} \widehat{\theta}^{n}\right\| .
\end{aligned}
$$


Therefore, using (4.37)-(4.39) in 4.36) and then multiplying the resulting inequality by $e^{-\alpha k}$, we obtain

$$
\begin{aligned}
\left\|\bar{\partial}_{t} \widehat{\theta}^{n}\right\|^{2}+\frac{1}{2} e^{-\alpha k}\left(1+\left\|\nabla U^{n}\right\|^{2}\right) \bar{\partial}_{t}\left\|\nabla \widehat{\theta}^{n}\right\|^{2} & \leq \frac{\left(1-e^{-\alpha k}\right)}{k}\left\|\widehat{\theta}^{n}\right\|\left\|\bar{\partial}_{t} \widehat{\theta}^{n}\right\|+e^{-\alpha k} e^{\alpha t_{n}}\left\|\bar{\partial}_{t} \eta^{n}\right\|\left\|\bar{\partial}_{t} \widehat{\theta}^{n}\right\| \\
& +e^{-\alpha k} e^{\alpha t_{n}}\left\|u_{t}\left(t_{n}\right)-\bar{\partial}_{t} u\left(t_{n}\right)\right\|\left\|\bar{\partial}_{t} \widehat{\theta}^{n}\right\| \\
& +C e^{-\alpha k} e^{\alpha t_{n}}\left\|\nabla u\left(t_{n}\right)\right\|\left\|\Delta u\left(t_{n}\right)\right\|\left\|\nabla \eta^{n}\right\|\left\|\bar{\partial}_{t} \widehat{\theta}^{n}\right\| \\
& +e^{-\alpha k} e^{\alpha t_{n}}\left(\left\|\nabla U^{n}\right\|+\left\|\nabla u\left(t_{n}\right)\right\|\right)\left\|\nabla \theta^{n}\right\|\left\|\Delta u\left(t_{n}\right)\right\|\left\|\bar{\partial}_{t} \widehat{\theta}^{n}\right\| .
\end{aligned}
$$

Apply Young's inequality $a b \leq \frac{\epsilon}{2} a^{2}+\frac{1}{2 \epsilon} b^{2}$ with $\epsilon=5$ and also $\frac{\left(1-e^{-\alpha k}\right)}{k}=\alpha e^{-\alpha k^{*}}$ for some $k^{*} \in(0, k)$ and then multiply the resulting inequality by 2 to obtain

$$
\begin{aligned}
\left\|\bar{\partial}_{t} \widehat{\theta}^{n}\right\|^{2}+e^{-\alpha k}\left(1+\left\|\nabla U^{n}\right\|^{2}\right) \bar{\partial}_{t}\left\|\nabla \widehat{\theta}^{n}\right\|^{2} & \leq C(\alpha)\left(e^{-2 \alpha k^{*}}\left\|\widehat{\theta}^{n}\right\|^{2}+e^{-2 \alpha k} e^{2 \alpha t_{n}}\left\|\bar{\partial}_{t} \eta^{n}\right\|^{2}\right. \\
& +e^{-2 \alpha k} e^{2 \alpha t_{n}}\left\|u_{t}\left(t_{n}\right)-\bar{\partial}_{t} u\left(t_{n}\right)\right\|^{2} \\
& +e^{-2 \alpha k} e^{2 \alpha t_{n}}\left\|\nabla u\left(t_{n}\right)\right\|^{2}\left\|\Delta u\left(t_{n}\right)\right\|^{2}\left\|\nabla \eta^{n}\right\|^{2} \\
& \left.+e^{-2 \alpha k} e^{2 \alpha t_{n}}\left(\left\|\nabla U^{n}\right\|^{2}+\left\|\nabla u\left(t_{n}\right)\right\|^{2}\right)\left\|\nabla \theta^{n}\right\|^{2}\left\|\Delta u\left(t_{n}\right)\right\|^{2}\right) .
\end{aligned}
$$

On multiplying (4.41) by $k e^{-2 \alpha t_{N}}$ and summing over $n=1$ to $N$, using (4.26), 4.28) and the estimate $\|\nabla \eta\|$ we arrive at

$$
\begin{aligned}
k e^{-2 \alpha t_{N}} \sum_{n=1}^{N} & \left\|\bar{\partial}_{t} \widehat{\theta}^{n}\right\|^{2}+e^{-\alpha k}\left(1+\left\|\nabla U^{N}\right\|^{2}\right)\left\|\nabla \theta^{N}\right\|^{2} \\
& \leq e^{-\alpha k} e^{-2 \alpha t_{N}}\left(1+\left\|\nabla U^{1}\right\|^{2}\right)\left\|\nabla \theta^{0}\right\|^{2}+e^{-\alpha k} e^{-2 \alpha t_{N}} k \sum_{n=1}^{N-1} \bar{\partial}_{t}\left\|\nabla U^{n+1}\right\|^{2}\left\|\nabla \widehat{\theta}^{n}\right\|^{2} \\
& +C(\alpha) e^{-2 \alpha t_{N}} k \sum_{n=1}^{N}\left\|\widehat{\theta}^{n}\right\|^{2}+C(\alpha) h^{2} e^{-2 \alpha t_{N}} \int_{0}^{t_{N}} e^{2 \alpha s}\left\|\nabla u_{t}(s)\right\|^{2} d s \\
& +C(\alpha) e^{-2 \alpha t_{N}} k^{2} \int_{0}^{t_{N}} e^{2 \alpha s}\left\|u_{t t}(s)\right\|^{2} d s \\
& +e^{-2 \alpha k} e^{-2 \alpha t_{N}} h^{2} k \sum_{n=1}^{N} e^{2 \alpha t_{n}}\left\|\nabla u\left(t_{n}\right)\right\|^{2}\left\|\Delta u\left(t_{n}\right)\right\|^{4} \\
& +e^{-2 \alpha k} e^{-2 \alpha t_{N}} k \sum_{n=1}^{N} e^{2 \alpha t_{n}}\left(\left\|U^{n}\right\|^{2}+\left\|u\left(t_{n}\right)\right\|^{2}\right)\left\|\Delta u\left(t_{n}\right)\right\|^{2}\left\|\nabla \theta^{n}\right\|^{2}
\end{aligned}
$$

From remark 4.1. Lemma $4.1 e^{-2 \alpha t_{N}}\left(k \sum_{n=1}^{N} e^{2 \alpha t_{n}}\right) \leq C$ and the previous theorem 4.3 , the first term, second term, third term and last term on the right hand side of equation 4.42 are bounded. From Lemmas 2.2, 2.4, 2.5. 2.7 the other terms on the right hand side of equation 4.42 are bounded. Therefore, we arrive at

$$
\begin{aligned}
& k e^{-2 \alpha t_{N}} \sum_{n=1}^{N}\left\|\bar{\partial}_{t} \widehat{\theta}^{n}\right\|^{2}+e^{-\alpha k}\left(1+\left\|\nabla U^{N}\right\|^{2}\right)\left\|\nabla \theta^{N}\right\|^{2} \\
& \leq C(\alpha, K) e^{-2 \alpha t_{N}}\left(h^{2}+k^{2}\right)\left(\left\|u_{0}\right\|_{H^{3}}^{2}+\left(\left(1+\left\|\nabla u_{0}\right\|^{2}\right)^{2}\left\|u_{0}\right\|_{H^{2}}^{2}+\left\|f_{0}\right\|^{2}\right)\right. \\
& \left.\quad+\int_{0}^{t_{N}} e^{2 \alpha s}\left\|f_{s}\right\|_{-1}^{2} d s\right) .
\end{aligned}
$$

This completes the rest of the proof. 
Since at each time level, we need to solve the system of nonlinear equation, below, we discuss modified backward Euler method which gives rise to a system of linear equations at each time step.

\subsection{Modified Backward Euler Method}

For $n \geq 1$ and given $U^{n-1}$, the fully discrete linear scheme based on backward Euler method is to seek $U^{n} \in V_{h}$ as a solution of

$$
\begin{aligned}
& \left(\bar{\partial}_{t} U^{n}, \varphi\right)+\left(1+\left\|\nabla U^{n-1}\right\|^{2}\right)\left(\nabla U^{n}, \nabla \varphi\right)=\left(f^{n}, \varphi\right) \quad \forall \varphi \in V_{h}, \\
& U^{0}=u_{0 h} .
\end{aligned}
$$

At each time level using a priori bound of $U^{n}$, this system of linear equation has a unique solution. Now for the error analysis, split the error $e^{n}=u\left(t_{n}\right)-U^{n}=\left(u\left(t_{n}\right)-\widetilde{u}\left(t_{n}\right)\right)-\left(U^{n}-\widetilde{u}\left(t_{n}\right)\right)=$ $\eta^{n}-\theta^{n}$, the equation in $\theta^{n}$ becomes

$$
\begin{aligned}
\left(\bar{\partial}_{t} \theta^{n}, \varphi_{h}\right)+\left(\left(1+\left\|\nabla U^{n-1}\right\|^{2}\right) \nabla U^{n}-\right. & \left.\left(1+\left\|\nabla \widetilde{u}\left(t_{n-1}\right)\right\|^{2}\right) \nabla \widetilde{u}\left(t_{n}\right), \nabla \varphi_{h}\right) \\
= & \left(\bar{\partial}_{t} \eta^{n}, \varphi_{h}\right)+\left(u_{t}\left(t_{n}\right)-\bar{\partial}_{t} u\left(t_{n}\right), \varphi_{h}\right) \\
& +\left(\left(1+\left\|\nabla u\left(t_{n}\right)\right\|^{2}\right) \nabla u\left(t_{n}\right)-\left(1+\left\|\nabla \widetilde{u}\left(t_{n-1}\right)\right\|^{2}\right) \nabla \widetilde{u}\left(t_{n}\right), \nabla \varphi_{h}\right) .
\end{aligned}
$$

Theorem 4.5. Assume that $0<\alpha<\frac{\lambda_{1}}{2}$ and choose $k_{0}>0$ such that for $0<k \leq k_{0} 4.13$ is true. Then, there exists a positive constant $C=C\left(\lambda_{1}, K_{1}\right)$ independent of $h$ and $k$ such that

$$
\begin{aligned}
\left\|\theta^{N}\right\|^{2}+ & k \beta e^{-2 \alpha t_{N}} \sum_{n=1}^{N} e^{2 \alpha t_{n}}\left\|\nabla \theta^{n}\right\|^{2} \\
\leq & C\left(\lambda_{1}, K_{1}\right) e^{-2 \alpha t_{N}}\left(k^{2}+h^{2}\right)\left(\left\|u_{0}\right\|_{H^{3}}^{2}+\left(\left(1+\left\|\nabla u_{0}\right\|^{2}\right)^{2}\left\|u_{0}\right\|_{H^{2}}^{2}+\left\|f_{0}\right\|^{2}\right)\right. \\
& \left.+\int_{0}^{t_{N}} e^{2 \alpha s}\left\|f_{s}\right\|_{-1}^{2} d s\right),
\end{aligned}
$$

where $\beta=\left(e^{-\alpha k}-\frac{2}{k \lambda_{1}}\left(1-e^{-\alpha k}\right)\right)>0$.

Proof. Multiplying 4.45) by $e^{\alpha t_{n}}$ and putting $\varphi_{h}=e^{\alpha t_{n}} \theta^{n}=\widehat{\theta}^{n}$, we obtain

$$
\begin{aligned}
\left(e^{\alpha t_{n}} \bar{\partial}_{t} \theta^{n}, \widehat{\theta}^{n}\right)+e^{\alpha t_{n}} & \left(\left(1+\left\|\nabla U^{n-1}\right\|^{2}\right) \nabla U^{n}-\left(1+\left\|\nabla \widetilde{u}\left(t_{n-1}\right)\right\|^{2}\right) \nabla \widetilde{u}\left(t_{n}\right), \nabla \widehat{\theta}^{n}\right) \\
& =e^{\alpha t_{n}}\left(\bar{\partial}_{t} \eta^{n}, \widehat{\theta}^{n}\right)+e^{\alpha t_{n}}\left(u_{t}\left(t_{n}\right)-\bar{\partial}_{t} u\left(t_{n}\right), \widehat{\theta}^{n}\right) \\
& +e^{\alpha t_{n}}\left(\left(1+\left\|\nabla u\left(t_{n}\right)\right\|^{2}\right) \nabla u\left(t_{n}\right)-\left(1+\left\|\nabla \widetilde{u}\left(t_{n-1}\right)\right\|^{2}\right) \nabla \widetilde{u}\left(t_{n}\right), \nabla \widehat{\theta}^{n}\right) .
\end{aligned}
$$

The second term on the left hand side can be written as

$$
\begin{aligned}
e^{2 \alpha t_{n}}\left(\left(1+\left\|\nabla U^{n-1}\right\|^{2}\right)\right. & \left.\nabla U^{n}-\left(1+\left\|\nabla \widetilde{u}\left(t_{n-1}\right)\right\|^{2}\right) \nabla \widetilde{u}\left(t_{n}\right), \nabla \theta^{n}\right) \\
& =e^{2 \alpha t_{n}}\left(\left(1+\left\|\nabla U^{n}\right\|^{2}\right) \nabla U^{n}-\left(1+\left\|\nabla \widetilde{u}\left(t_{n}\right)\right\|^{2}\right) \nabla \widetilde{u}\left(t_{n}\right), \nabla \theta^{n}\right) \\
& +e^{\alpha t_{n}}\left(\left(\left\|\nabla U^{n-1}\right\|^{2}-\left\|\nabla U^{n}\right\|^{2}\right) \nabla U^{n}, \nabla \widehat{\theta}^{n}\right) \\
& +e^{\alpha t_{n}}\left(\left(\left\|\nabla \widetilde{u}\left(t_{n}\right)\right\|^{2}-\left\|\nabla \widetilde{u}\left(t_{n-1}\right)\right\|^{2}\right) \nabla \widetilde{u}\left(t_{n}\right), \nabla \widehat{\theta}^{n}\right) \\
& \geq\left\|\nabla \widehat{\theta}^{n}\right\|^{2}-C e^{2 \alpha t_{n}} k^{2}\left(\bar{\partial}_{t}\left\|\nabla U^{n}\right\|^{2}\right)^{2}\left\|\nabla U^{n}\right\|^{2}-\frac{1}{12}\left\|\nabla \widehat{\theta}^{n}\right\|^{2} \\
& -e^{\alpha t_{n}}\left(\left(\left\|\nabla \widetilde{u}\left(t_{n}\right)\right\|^{2}-\left\|\nabla \widetilde{u}\left(t_{n-1}\right)\right\|^{2}\right) \nabla \widetilde{u}\left(t_{n}\right),-\nabla \widetilde{\theta}^{n}\right) .
\end{aligned}
$$


Note that

$$
\begin{aligned}
\left\|\nabla \widetilde{u}\left(t_{n}\right)-\nabla \widetilde{u}\left(t_{n-1}\right)\right\|^{2} & =\left\|\int_{t_{n-1}}^{t_{n}}(\nabla \widetilde{u}(t))_{t} d t\right\|^{2} \\
& \leq C\left(k \int_{t_{n-1}}^{t_{n}}\left\|\nabla u_{t}\right\|^{2} d t+h^{2} k \int_{t_{n-1}}^{t_{n}}\left\|\Delta u_{t}\right\|^{2} d t\right) .
\end{aligned}
$$

The third term on the right hand side is bounded by

$$
\begin{aligned}
e^{\alpha t_{n}}\left(\left(1+\left\|\nabla u\left(t_{n}\right)\right\|^{2}\right) \nabla u\left(t_{n}\right)-\right. & \left.\left(1+\left\|\nabla \widetilde{u}\left(t_{n-1}\right)\right\|^{2}\right) \nabla \widetilde{u}\left(t_{n}\right), \nabla \widehat{\theta}^{n}\right) \\
& =e^{\alpha t_{n}}\left(\left(\left\|\nabla u\left(t_{n}\right)\right\|^{2}-\left\|\nabla \widetilde{u}\left(t_{n}\right)\right\|^{2}\right) \nabla u\left(t_{n}\right), \nabla \widehat{\theta}^{n}\right) \\
& +e^{\alpha t_{n}}\left(\left(\left\|\nabla \widetilde{u}\left(t_{n}\right)\right\|^{2}-\left\|\nabla \widetilde{u}\left(t_{n-1}\right)\right\|^{2}\right) \nabla u\left(t_{n}\right), \nabla \widehat{\theta}^{n}\right) \\
\leq & C e^{2 \alpha t_{n}} h^{2}\left\|\nabla u\left(t_{n}\right)\right\|^{4}\|\Delta u\|^{2}+\frac{1}{6}\left\|\nabla \widehat{\theta}^{n}\right\|^{2} \\
& +\left(C e^{2 \alpha t_{n}}\left(\left\|\nabla u\left(t_{n}\right)\right\|^{2}+\left\|\nabla u\left(t_{n-1}\right)\right\|^{2}\right)\left\|\nabla u\left(t_{n}\right)\right\|^{2}\right. \\
& \left.\left(k \int_{t_{n-1}}^{t_{n}}\left\|\nabla u_{t}\right\|^{2} d t+h^{2} k \int_{t_{n-1}}^{t_{n}}\left\|\Delta u_{t}\right\|^{2} d t\right)\right) .
\end{aligned}
$$

Now, continuing as before in Theorm 4.3 we arrive at

$\bar{\partial}_{t}\left\|\hat{\theta}^{n}\right\|^{2}+\left(e^{-\alpha k}-\frac{2}{k \lambda_{1}}\left(1-e^{-\alpha k}\right)\right)\left\|\nabla \hat{\theta}^{n}\right\|^{2} \leq \frac{3}{2 \lambda_{1}} e^{-\alpha k} e^{2 \alpha t_{n}}\left(\left\|\bar{\partial}_{t} \eta^{n}\right\|^{2}+\left\|u_{t}\left(t_{n}\right)-\bar{\partial}_{t} u\left(t_{n}\right)\right\|^{2}\right)$

$$
\begin{aligned}
& +C e^{2 \alpha t_{n}} k^{2}\left(\bar{\partial}_{t}\left\|\nabla U^{n}\right\|^{2}\right)^{2}\left\|\nabla U^{n}\right\|^{2}+C e^{2 \alpha t_{n}} h^{2}\left\|\nabla u\left(t_{n}\right)\right\|^{4}\|\Delta u\|^{2} \\
& +\frac{1}{6}\left\|\nabla \widehat{\theta}^{n}\right\|^{2}+\left(C e^{2 \alpha t_{n}}\left(\left\|\nabla u\left(t_{n}\right)\right\|^{2}+\left\|\nabla u\left(t_{n-1}\right)\right\|^{2}\right)\left\|\nabla u\left(t_{n}\right)\right\|^{2}\right. \\
& \left.\left(k \int_{t_{n-1}}^{t_{n}}\left\|\nabla u_{t}\right\|^{2} d t+h^{2} k \int_{t_{n-1}}^{t_{n}}\left\|\Delta u_{t}\right\|^{2} d t\right)\right) .
\end{aligned}
$$

Mutiply 4.51) by $k$, and summing over $n=1$ to $N$, we arrive at

$$
\begin{aligned}
\left\|\widehat{\theta}^{N}\right\|^{2}+k \beta \sum_{n=1}^{N}\left\|\nabla \widehat{\theta}^{n}\right\|^{2} & \leq\left\|\theta^{0}\right\|^{2}+C\left(\lambda_{1}\right)\left(h^{2}+k^{2}\right) \int_{0}^{t_{N}} e^{2 \alpha s}\left\|\nabla u_{t}(s)\right\|^{2} d s+C\left(\lambda_{1}\right) k^{2} \int_{0}^{t_{N}} e^{2 \alpha s}\left\|u_{t t}(s)\right\|^{2} d s \\
& +C\left(\lambda_{1}\right) h^{2} k^{2} \int_{0}^{t_{N}} e^{2 \alpha s}\left\|\Delta u_{t}(s)\right\|^{2} d s+C\left(K_{1}\right) k^{2} e^{-\alpha k}\left\|f^{n}\right\|_{L^{\infty}\left(L^{2}\right)}^{2}\left(k \sum_{n=1}^{N} e^{2 \alpha t_{n}}\right) .
\end{aligned}
$$

Proceed as in theorm 4.3 to complete the rest of the proof.

Theorem 4.6. Assume that $0<\alpha<\frac{\lambda_{1}}{2}$ and choose $k_{0}>0$ be such that for $0<k \leq k_{0}, 4.13$ is true. Then, there exists a positive constant $C=C(\alpha, K)$ holds

$$
\begin{aligned}
k e^{-2 \alpha t_{N}} \sum_{n=1}^{N} & \left\|\bar{\partial}_{t} \widehat{\theta}^{n}\right\|^{2}+e^{-\alpha k}\left(1+\left\|\nabla U^{N-1}\right\|^{2}\right)\left\|\nabla \theta^{N}\right\|^{2} \\
& \leq C\left(\alpha, K_{1}\right) e^{-2 \alpha t_{N}}\left(h^{2}+k^{2}\right)\left(\left\|u_{0}\right\|_{H^{3}}^{2}+\left(\left(1+\left\|\nabla u_{0}\right\|^{2}\right)^{2}\left\|u_{0}\right\|_{H^{2}}^{2}+\left\|f_{0}\right\|^{2}\right)\right. \\
& \left.+\int_{0}^{t_{N}} e^{2 \alpha s}\left\|f_{s}\right\|_{-1}^{2} d s\right),
\end{aligned}
$$

where $\beta=\left(e^{-\alpha k}-\frac{2}{k \lambda_{1}}\left(1-e^{-\alpha k}\right)\right)>0$. 
Proof. Multiply the equation 4.45 by $e^{\alpha t_{n}}$ and then putting $\varphi_{h}=\bar{\partial}_{t} \widehat{\theta}^{n}$, we obtain

$$
\begin{aligned}
e^{\alpha k}\left\|\bar{\partial}_{t} \widehat{\theta}^{n}\right\|^{2}+e^{\alpha t_{n}} & \left(\left(1+\left\|\nabla U^{n-1}\right\|^{2}\right) \nabla U^{n}-\left(1+\left\|\nabla \widetilde{u}\left(t_{n-1}\right)\right\|^{2}\right) \nabla \widetilde{u}\left(t_{n}\right), \nabla \bar{\partial}_{t} \widehat{\theta}^{n}\right) \\
& =\frac{\left(e^{\alpha k}-1\right)}{k}\left(\widehat{\theta}^{n}, \bar{\partial}_{t} \widehat{\theta}^{n}\right)+e^{\alpha t_{n}}\left(\bar{\partial}_{t} \eta^{n}, \bar{\partial}_{t} \widehat{\theta}^{n}\right)+e^{\alpha t_{n}}\left(u_{t}\left(t_{n}\right)-\bar{\partial}_{t} u\left(t_{n}\right), \bar{\partial}_{t} \widehat{\theta}^{n}\right) \\
& +e^{2 \alpha t_{n}}\left(\left(1+\left\|\nabla u\left(t_{n}\right)\right\|^{2}\right) \nabla u\left(t_{n}\right)-\left(1+\left\|\nabla \widetilde{u}\left(t_{n-1}\right)\right\|^{2}\right) \nabla \widetilde{u}\left(t_{n}\right), \nabla \bar{\partial}_{t} \widehat{\theta}^{n}\right) .
\end{aligned}
$$

The second term on the left hand side of 4.54, can be bounde by

$$
\begin{aligned}
e^{\alpha t_{n}}\left(\left(1+\left\|\nabla U^{n-1}\right\|^{2}\right) \nabla U^{n}-\right. & \left.\left(1+\left\|\nabla \widetilde{u}\left(t_{n-1}\right)\right\|^{2}\right) \nabla \widetilde{u}\left(t_{n}\right), \nabla \bar{\partial}_{t} \widehat{\theta}^{n}\right) \\
& =\left(1+\left\|\nabla U^{n-1}\right\|^{2}\right)\left(\nabla \widehat{\theta}^{n}, \nabla \bar{\partial}_{t} \widehat{\theta}^{n}\right) \\
& -e^{\alpha t_{n}}\left(\left(\left\|\nabla U^{n-1}\right\|^{2}-\left\|\nabla \widetilde{u}\left(t_{n-1}\right)\right\|^{2}\right) \Delta u\left(t_{n}\right), \bar{\partial}_{t} \widehat{\theta}^{n}\right) .
\end{aligned}
$$

The fourth term on the right hand side is bounded by

$$
\begin{aligned}
e^{\alpha t_{n}}\left(\left(1+\left\|\nabla u\left(t_{n}\right)\right\|^{2}\right) \nabla u\left(t_{n}\right)-\right. & \left.\left(1+\left\|\nabla \widetilde{u}\left(t_{n}\right)\right\|^{2}\right) \nabla \widetilde{u}\left(t_{n}\right), \nabla \bar{\partial}_{t} \widehat{\theta}^{n}\right) \\
& =-e^{\alpha t_{n}}\left(\left(\left\|\nabla u\left(t_{n}\right)\right\|^{2}-\left\|\nabla \widetilde{u}\left(t_{n}\right)\right\|^{2}\right) \Delta u\left(t_{n}\right), \bar{\partial}_{t} \widehat{\theta}^{n}\right) \\
& -e^{\alpha t_{n}}\left(\left(\left\|\nabla \widetilde{u}\left(t_{n}\right)\right\|^{2}-\left\|\nabla \widetilde{u}\left(t_{n-1}\right)\right\|^{2}\right) \Delta u\left(t_{n}\right), \bar{\partial}_{t} \widehat{\theta}^{n}\right) \\
& \leq C h e^{\alpha t_{n}}\left\|\nabla u\left(t_{n}\right)\right\|\left\|\Delta u\left(t_{n}\right)\right\|^{2}\left\|\bar{\partial}_{t} \widehat{\theta}^{n}\right\| \\
& +e^{\alpha t_{n}}\left(\left\|\nabla u\left(t_{n}\right)\right\|+\left\|\nabla u\left(t_{n-1}\right)\right\|\right)\left(\left\|\nabla \widetilde{u}\left(t_{n}\right)-\nabla \widetilde{u}\left(t_{n-1}\right)\right\|\right)\left\|\Delta u\left(t_{n}\right)\right\|\left\|\bar{\partial}_{t} \widehat{\theta}^{n}\right\| .
\end{aligned}
$$

Therefore proceeding as in theorem 4.4 we obtain

$$
\begin{aligned}
\left\|\bar{\partial}_{t} \widehat{\theta}^{n}\right\|^{2}+e^{-\alpha k}\left(1+\left\|\nabla U^{n-1}\right\|^{2}\right) \bar{\partial}_{t}\left\|\nabla \widehat{\theta}^{n}\right\|^{2} & \leq C(\alpha)\left(e^{-2 \alpha k^{*}}\left\|\widehat{\theta}^{n}\right\|^{2}+e^{-2 \alpha k} e^{2 \alpha t_{n}}\left\|\bar{\partial}_{t} \eta^{n}\right\|^{2}\right. \\
& +e^{-2 \alpha k} e^{2 \alpha t_{n}}\left\|u_{t}\left(t_{n}\right)-\bar{\partial}_{t} u\left(t_{n}\right)\right\|^{2} \\
& +e^{-2 \alpha k}\left(\left\|\nabla U^{n-1}\right\|^{2}+\left\|\nabla u\left(t_{n-1}\right)\right\|^{2}\right)\left\|\Delta u\left(t_{n}\right)\right\|^{2}\left\|\nabla \theta^{n-1}\right\|^{2} \\
& +h^{2} e^{-2 \alpha k} e^{2 \alpha t_{n}}\left\|\nabla u\left(t_{n}\right)\right\|^{2}\left\|\Delta u\left(t_{n}\right)\right\|^{4} \\
& +\left(e^{-2 \alpha k} e^{2 \alpha t_{n}}\left(\left\|\nabla u\left(t_{n}\right)\right\|^{2}+\left\|\nabla u\left(t_{n-1}\right)\right\|^{2}\right)\right. \\
& \left.\left.\left(\left\|\nabla \widetilde{u}\left(t_{n}\right)-\nabla \widetilde{u}\left(t_{n-1}\right)\right\|\right)\left\|\Delta u\left(t_{n}\right)\right\|\right)\right) .
\end{aligned}
$$

The rest of the proof is same as in Theorem 4.4 which also uses the estimate in Theorem 4.5 . This completes the rest of the proof.

\section{$5 \quad$ Numerical Experiment}

In this section, we discuss fully discrete finite element formulation of (1.1)-(1.3) using modified backward Euler method. Now time variable is discritized by replacing the time derivative by difference quotient. Let $k$ be the time step and $U^{n}$ be the approximation of $u(t)$ in $V_{h}$ at $t=t_{n}=$ $n k$. We now apply modified backward Euler approximation to (3.1).

Example 5.1. Here, we choose the right hand side function $f$ in such a way so that the exact solution is $u=x(1-x) y(1-y) e^{-t}$ in $\Omega=(0,1) \times(0,1)$ and time $t=[0,1]$, which satisfy the Dirichlet boundary condition.

In Table 1, the convergence rates are given for $t=1$. Observe that $\left\|\nabla u^{n}-\nabla U^{n}\right\|$ is of order one 
Table 1: Errors and convergence rate for modified backward Euler method

\begin{tabular}{ccccc}
\hline$h$ & $\left\|u\left(t_{n}\right)-U^{n}\right\|_{L^{2}}$ & Conv. Rate & $\left\|u\left(t_{n}\right)-U^{n}\right\|_{H^{1}}$ & Conv. Rate \\
\hline \hline$\frac{1}{2}$ & 0.002493 & & 0.010547 & \\
\hline$\frac{1}{4}$ & 0.000715 & 1.801077 & 0.006027 & 0.807341 \\
\hline$\frac{1}{8}$ & 0.000287 & 1.931655 & 0.003242 & 0.931121 \\
\hline$\frac{1}{16}$ & 0.000048 & 1.956815 & 0.001605 & 0.977676 \\
\hline$\frac{1}{32}$ & 0.000012 & 1.904400 & 0.000806 & 0.993114 \\
\hline
\end{tabular}

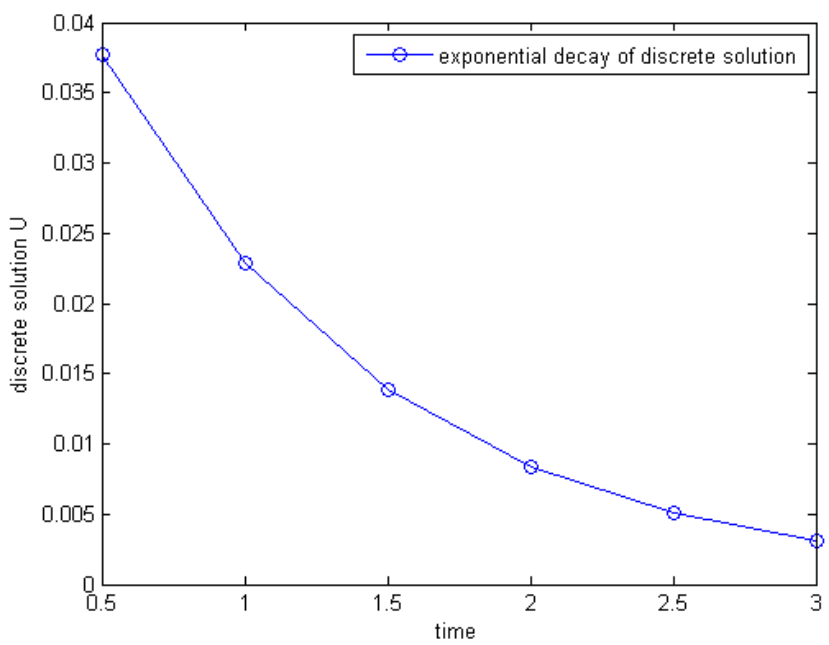

Figure 1: exponential decay of solution

as predicted by the theory. It is also observed numerically that the convergence rate for $L^{2}$ - error is of order 2, but we still do not have a theory to back this claim.

Since $f=O\left(e^{-t}\right)$, it is further observed in Fig 1 that discrete solution $\|U\|_{L^{\infty}\left(H^{1}\right)}$ decays exponentially as predicted by the theory.

Example 5.2. Here, we choose the right hand side function $f$ in such a way so that the exact solution is $u=t \sin (\pi x) \sin (\pi y)$ in $\Omega=(0,1) \times(0,1)$ and time $t=[0,1]$, which satisfy the Dirichlet boundary condition.

In Table 2, the convergence rates are given for $t=1$. Observe that $\left\|\nabla u^{n}-\nabla U^{n}\right\|$ is of order one as predicted by the theory.

Table 2: Errors and convergence rate for modified backward Euler method

\begin{tabular}{ccccc}
\hline$h$ & $\left\|u\left(t_{n}\right)-U^{n}\right\|_{L^{2}}$ & Conv. Rate & $\left\|u\left(t_{n}\right)-U^{n}\right\|_{H^{1}}$ & Conv. Rate \\
\hline \hline$\frac{1}{2}$ & 0.091805 & & 0.470744 & \\
\hline$\frac{1}{4}$ & 0.024631 & 1.898054 & 0.268189 & 0.811695 \\
\hline$\frac{1}{8}$ & 0.006364 & 1.952374 & 0.141461 & 0.922836 \\
\hline$\frac{1}{16}$ & 0.001576 & 2.013456 & 0.072073 & 0.972865 \\
\hline$\frac{1}{32}$ & 0.000358 & 2.135151 & 0.036255 & 0.991270 \\
\hline
\end{tabular}




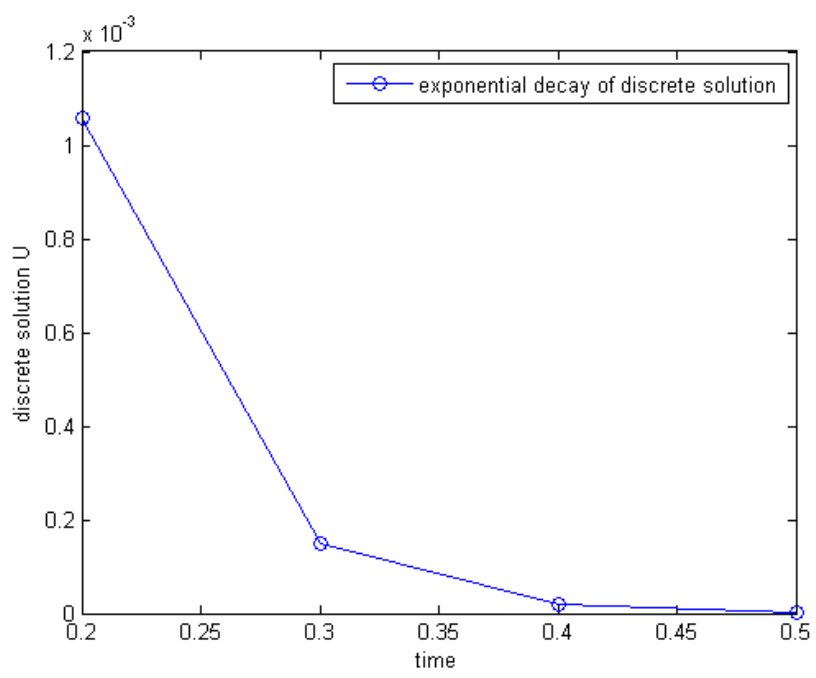

Figure 2: Exponential decay of solution when $\mathrm{f}=0$

Example 5.3. Now in this example we have taken right hand side $f=0$. We do not know the exact form of exact solution. We have chosen $u_{0}$ (exact solution at $\left.t=0\right)$ as $u_{0}=x(1-x) y(1-$ $y) \sin (x+y)$.

Here we have observed in Fig 2 that discrete solution decays exponentially as time increase as predicted by the theory when $f=0$.

\section{References}

[1] Alves, C. O. and Correa, J. S. A.: On existence of solutions for a class of problem involving nonlinear operator, Comm. Appl. Nonlinear Anal. 8 (2001), pp. 43-56.

[2] Chang, N. H. and Chipot, M.: Nonlinear nonlocal evolution problems, RACSAM, Rev. R. Acad. Cien. Serie A. Mat, 97(2003), pp. 393-415.

[3] Chipot M. Elements of Nonlinear Analysis, Birkhauser Advanced Text North-Holland (2000).

[4] Chipot, M. and Lovat, B.: Existence and uniqueness results for a class of nonlocal elliptic and parabolic problems, DCDIS. Series A, 8(2001), pp. 35-51.

[5] Chipot, M.: Valente, V., and Caffarelli, G. V.Remarks on a nonlocal problem involving the Dirichlet energy, Rend. Sem. Mat. Univ. Padova, 110 (2003), pp. 199-220.

[6] Gudi, T.: Finite element method for a nonlocal problem of Kirchhoff type, SIAM J. Numer. Anal, 50(2012), pp. 657-668.

[7] Kesavan, S.: Topics in Functional Analysis and Application, New Age International (P)Ltd, Publishers, 2008.

[8] Thomee, V. Galerkin Finite Element Methods for Parabolic Problems, Springer, 1997.

[9] Zheng, S. and Chipot, M.: Asymptotic behavior of solutions to nonlinear parabolic equations with nonlocal terms, Asymptot. Anal. 45 (2005), pp. 301-312. 